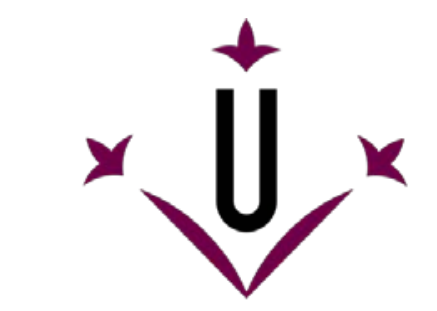

Universitat de Lleida

Document downloaded from:

http://hdl.handle.net/10459.1/67895

The final publication is available at:

https://doi.org/10.1007/s40879-018-0304-3

Copyright

(c) Springer, 2019 


\title{
CYCLICITY OF NILPOTENT CENTERS WITH MINIMUM ANDREEV NUMBER
}

\author{
ISAAC A. GARCÍA
}

\begin{abstract}
We consider polynomial families of real planar vector fields for which the origin is a monodromic nilpotent singularity having minimum Andree's number. There the centers are characterized by the existence of a formal inverse integrating factor. For such families we give, under some assumptions, global bounds on the maximum number of limit cycles that can bifurcate from the singularity under perturbations within the family.
\end{abstract}

\section{INTRODUCTION}

A singular point of an analytic planar vector field is nilpotent when the linearization around it does not vanish but the associated eigenvalues do. By an affine change of coordinates and a time rescaling an analytic planar vector field with a nilpotent singularity can be placed in the form

$$
\dot{x}=y+P(x, y), \quad \dot{y}=Q(x, y),
$$

where $P$ and $Q$ are analytic functions near the origin without constant or linear terms. In this work we focus on the monodromic case for which the local flow of (1) near the origin rotate about it. It is well known that, in this case, the local qualitative phase portrait corresponds to either a center or a focus. You can see two independent proofs of this fact in [14] and [24]. Andreev in [6] characterizes analytic systems (1) for which the origin is monodromic. This characterization depends on two positive integer numbers $(n, \hat{\beta}) \in \mathbb{N}^{2}$ with $n \geq 2$ and $\hat{\beta} \geq n-1$, see the forthcoming Theorem 22. We call $n$ the Andreev number associated to an analytic monodromic nilpotent singularity which is invariant under analytic orbital equivalency, see [16].

From now we restrict our selves to families in the class of monodromic nilpotent singularities with minimum Andreev number $(n, \hat{\beta})=(2,1)$, that is, those that fall in case (iii) of Theorem 22. This monodromic family is characterized by the following form:

$$
\dot{x}=y+a x^{2}+P^{*}(x, y ; \zeta), \quad \dot{y}=b x^{3}+c x y+Q^{*}(x, y ; \zeta),
$$

where the parameter restrictions $b-a c<0,2 a+c \neq 0$ and $8 b+(c-2 a)^{2}<0$ holds by monodromy and $P^{*}$ and $Q^{*}$ denote $(1,2)$-quasihomogeneous higher order terms in $x$ and $y$ with vector parameter $\zeta$.

2010 Mathematics Subject Classification. 37G15, 37G10, 34C25.

Key words and phrases. Monodromic singularity, nilpotent center, cyclicity, inverse integrating factor.

The author is partially supported by a MINECO grant number MTM2017-84383-P and an AGAUR grant number 2017SGR-1276. 
Following Andreev's ideas [6], see $\S 3.2$, any system (1) with a monodromic singularity at the origin with $(n, \hat{\beta})=(2,1)$ is analytically conjugate to the Andreev's canonical form

$$
\dot{x}=-y+y \tilde{P}(x, y ; \nu), \quad \dot{y}=x^{3}+\omega x y+\tilde{Q}(x, y ; \nu),
$$

where $\tilde{P}$ and $\tilde{Q}$ denote terms with $(1,2)$-quasihomogeneous degrees greater than 0 and 3 , respectively, and with parameter space $\mathcal{E}=\left\{(\omega, \nu) \in \Omega \times \mathbb{R}^{p}\right\}$ and

$$
\Omega=\left\{\omega \in \mathbb{R}^{*}: \omega^{2}-8<0\right\}
$$

Therefore we see that $\omega$ is a special parameter because it appears in the the $(1,2)$ quasihomogeneous truncation of minimum degree of the vector field (3).

In what follows we will denote by $\mathcal{X}$ and $\hat{\mathcal{X}}$ the vector fields associated to (1) (or (2) depending the context) and (3), respectively. Hence $\mathcal{X}$ is the linear differential operator $\mathcal{X}=\left(y+P(x, y) \partial_{x}+Q(x, y) \partial_{y}\right.$ and $\operatorname{div}(\mathcal{X})$ its divergence.

It is well known that nilpotent centers, unlike the non-degenerate centers with associated non-vanishing pure imaginary eigenvalues, are not characterized by the existence of a formal first integral. Some exceptions are the trivial Hamiltonian centers $\dot{x}=\partial_{y} H(x, y), \dot{y}=-\partial_{x} H(x, y)$, the monodromic time-reversible with respect to $(x, y, t) \mapsto(x,-y, t)$ (see [8]) or the monodromic $\mathbb{Z}_{2}$-symmetric (those invariant under $(x, y) \mapsto(-x,-y))$, see [2]. Notice that these exceptions may happen in a general system (1) but never occurs in systems (2) or (3) for which $(n, \hat{\beta})=(2,1)$.

However, nilpotent centers with a minimum Andreev number $n=2$ are characterized by the existence of a formal inverse integrating factor. This last sentence follows joining the three works [26], [27] and [28] although a simpler and unified different proof can be found in [16]. We remark that the nilpotent monodromic class $(n, \hat{\beta})=(2,1)$ is not analyzed in [3], and is out of the range of applicability of the families studied in [21] and [17].

It is known that a formal Lyapunov function exists for any non-degenerate monodromic singularity, giving rise to the so-called focal quantities, which are nothing more than the obstructions to the formal integrability. In an analogous way, for the class of monodromic nilpotent singularities with $(n, \hat{\beta})=(2,1)$ (and in particular for family (3)), we can found the obstructions $\hat{\eta}_{j}(\omega, \nu)$ to the existence of an inverse formal integrating factor by means of an algorithm that only involves algebraic calculations.

We call $\hat{\eta}_{j}(\omega, \nu)$ the integrability focus quantities of a polynomial family (3) parameterized by its coefficients. Then it can be checked that $\left\{\hat{\eta}_{j}(\omega, \nu)\right\}_{j \in \mathbb{N}} \subset$ $\mathbb{R}(\omega)[\nu]$. Here, $\mathbb{R}(\omega)[\nu]$ denotes the set of polynomials in $\nu$ with rational coefficients in $\omega$. Since a polynomial ring over a field is Noetherian and the elements of $\mathbb{R}(\omega)$ are just formal expressions so it is a field, hence $\mathbb{R}(\omega)[\nu]$ is a Noetherian ring. System (3) with $(\omega, \nu)=\left(\omega^{*}, \nu^{*}\right) \in \mathcal{E}$ has a center at the origin if and only if $\hat{\eta}_{j}\left(\omega^{*}, \nu^{*}\right)=0$ for any $j \in \mathbb{N}$, in which case there is a formal inverse integrating factor of (3).

On the other hand, using adequate coordinates, we find the Poincaré return map $\Pi: \Sigma \subseteq \mathbb{R} \rightarrow \mathbb{R}$ defined by an analytic diffeomorphism on a transversal section $\Sigma$ to the flow with endpoint at the nilpotent singularity. More precisely, using ideas of Lyapunov [29] we can parameterize $\Sigma$ such that $\Pi(r)=r+\sum_{j \geq 2} v_{j}(\omega, \nu) r^{j}$ where the elements of the sequence $\left\{v_{j}(\omega, \nu)\right\}_{j \in \mathbb{N}} \subset \mathbb{R}(\omega)[\nu]$ are called Poincaré-Lyapunov quantities. Both sequences of integrability focus quantities and Poincaré-Lyapunov quantities share the property that system $(3)$ with $(\omega, \nu)=\left(\omega^{*}, \nu^{*}\right) \in \mathcal{E}$ has a 
nilpotent center at the origin if and only if each element in both sequences vanishes at $\left(\omega^{*}, \nu^{*}\right)$. We want to stress here that the integrability focus quantities are much easier to work with than the Poincaré-Lyapunov quantities. The reason for this to happen is because the $\hat{\eta}_{j}$ are computed via an algebraic algorithmic easy to use with a computer algebra system while the calculation of the $v_{j}$ needs quadratures of Lyapunov generalized trigonometric functions, see for example the computations in [4] and some of the results in [21].

Nonetheless we will relate both sequences $\left\{\hat{\eta}_{j}(\omega, \nu)\right\}_{j \in \mathbb{N}}$ and $\left\{v_{j}(\omega, \nu)\right\}_{j \in \mathbb{N}}$ by means of computational algebra techniques in such a way that we can in some cases bound the cyclicity of centers at the origin of polynomial families (3). Recall that the cyclicity of the nilpotent monodromic singularity at the origin of system (3) with $(\omega, \nu)=\left(\omega^{\dagger}, \nu^{\dagger}\right) \in \mathcal{E}$ is the maximum number of limit cycles that can bifurcate from it for parameters $(\omega, \nu) \in \mathcal{E}$ such that $\left\|(\omega, \nu)-\left(\omega^{\dagger}, \nu^{\dagger}\right)\right\| \ll 1$.

Although there are in the literature studies on the cyclicity of nilpotent focus, the cyclicity of centers is harder to analyze. The main reason is that Hopf bifurcation from foci is a finite-codimension bifurcation in contrast with the center bifurcation. In some papers like in [33] the Hopf bifurcation at nilpotent center is analyzed under the restriction that the family is close to a Hamiltonian center by introducing a small perturbation parameter. Notice that this restriction is never satisfied by our systems (2) or (3). As far as I know this is the first work where a general method for computing global upper bound on the nilpotent center cyclicity is obtained for the monodromic class $(n, \hat{\beta})=(2,1)$. By the way we will observe the troublesome role that the parameter $\omega$ plays, particularly when we need to complexify the parameter space in order to analyze the cyclicity problem. This kind of problems are very similar to those encountered in the paper [20] related to the cyclicity of nondegenerate centers on center manifolds for 3-dimensional systems.

\section{Main Results}

Our first result is in fact the definition of integrability focus quantities $\hat{\eta}_{j}(\omega, \nu)$ associated to Andreev's canonical family (3).

Theorem 1. Let $\hat{\mathcal{X}}$ be the vector field associated to the Andreev's canonical form (3). Then, there is a unique formal series $W(x, y)=\sum_{k \geq 4} W_{k}(x, y)$ where $W_{k}$ are $(1,2)$-quasihomogeneous polynomials of weighted degree $k$ with leading term $W_{4}(x, y)=x^{4}+2 y^{2}+\omega x^{2} y$ and such that

$$
\hat{\mathcal{X}}(W)-W \operatorname{div}(\hat{\mathcal{X}})=x^{4} \sum_{j \geq 1} \hat{\eta}_{j}(\omega, \nu) x^{2 j} .
$$

Moreover, for any polynomial family (3) parameterized by its coefficients, we have $\hat{\eta}_{j}(\omega, \nu) \in \mathbb{R}(\omega)[\nu]$ and they are well defined for $\omega \in \Omega$.

The next result shows the relationship between the $v_{j}(\omega, \nu)$ and the $\hat{\eta}_{j}(\omega, \nu)$ quantities of system (3). Statement (i) was already proved in a more general context in [19] and we write it only for the sake of completeness.

Theorem 2. Consider a polynomial Andreev canonical family (3) and let $v_{k}(\omega, \nu)$ be the Poincaré-Lyapunov quantities and $\hat{\eta}_{k}(\omega, \nu)$ the integrating focal quantities associated to it. Then there exist positive real numbers $\alpha_{k}$ such that:

(i) If $v_{2 k}=0$ then $v_{2 k+1}=0$ for any $k \geq 1$;

(ii) $v_{2}=\alpha_{1} \hat{\eta}_{1}$; 
(iii) If $\hat{\eta}_{1}=\cdots=\hat{\eta}_{k-1}=0$ then $v_{2 k}=\alpha_{k} \hat{\eta}_{k}$ for any $k \geq 2$.

We will use from now the following standard algebraic notation. The ideal generated by elements $r_{1}, \ldots, r_{s}$ of a ring $R$ will be denoted by $\left\langle r_{1}, \ldots, r_{s}\right\rangle$. For a field $\mathbb{K}$ the variety in $\mathbb{K}^{n}$ of an ideal $I \subset \mathbb{K}\left[x_{1}, \ldots, x_{n}\right]$ will be denoted $\mathbf{V}_{\mathbb{K}}(I)$. In this work we will only use either $\mathbb{K}=\mathbb{R}$ or $\mathbb{K}=\mathbb{C}$.

Definition 3. We define the Bautin ideal $\mathcal{B}$ associated with a polynomial Andreev canonical family $(3)$ as the ideal $\mathcal{B}=\left\langle v_{j}(\omega, \nu): j \in \mathbb{N}\right\rangle$ in the Noetherian ring $\mathbb{R}(\omega)[\nu]$. Also, we denote by $\mathcal{B}_{2 k}$ the ideal $\mathcal{B}_{2 k}=\left\langle v_{2}, v_{4}, \ldots, v_{2 k}\right\rangle$. We define analogously the ideals $\hat{\mathcal{I}}=\left\langle\hat{\eta}_{j}(\omega, \nu): j \in \mathbb{N}\right\rangle$ in the ring $\mathbb{R}(\omega)[\nu]$ and $\hat{\mathcal{I}}_{k}=\left\langle\hat{\eta}_{1}, \hat{\eta}_{2}, \ldots, \hat{\eta}_{k}\right\rangle$.

The next result is a corollary of Theorem 2 .

Corollary 4. The relations $v_{2 k+j} \in \hat{\mathcal{I}}_{k}$ for $j \in\{0,1\}$ and any $k \geq 1$ hold. In particular $\hat{\mathcal{I}}_{k}=\mathcal{B}_{2 k}$ and $\hat{\mathcal{I}}=\mathcal{B}$.

2.1. The Bautin ideal and the cyclicity of the nilpotent singularity. We define the displacement map $\delta$ associated to family (3) as

$$
\delta\left(r_{0} ; \omega, \nu\right)=\Pi\left(r_{0} ; \omega, \nu\right)-r_{0},
$$

where $\Pi$ is the Poincaré return map associated to the origin of (3) which is computed using the parameterization of a transversal section to the flow as it is detailed in §3.3. So the fixed points of $\Pi$ become zeroes of $\delta$ and correspond with the small amplitude periodic orbits of (3). Now we are going to perform a Bautin-type analysis [7] (see [31] for a modern exposition) to get upper bounds on the number of positive roots of $\delta(. ; \omega, \nu)$ close to the origin.

Assume first that system (3) with $(\omega, \nu)=\left(\omega^{\dagger}, \nu^{\dagger}\right) \in \Omega \times \mathbb{R}^{p}$ possesses a focus at the origin. Then there is a positive integer $k$ called the order of the focus such that $v_{j}\left(\omega^{\dagger}, \nu^{\dagger}\right)=0$ for $j \leqslant 2 k-1$ but $v_{2 k}\left(\omega^{\dagger}, \nu^{\dagger}\right) \neq 0$. In this situation, for $(\omega, \nu)$ sufficiently close to $\left(\omega^{\dagger}, \nu^{\dagger}\right)$ we have $v_{2 k}(\omega, \nu) \neq 0$ by continuity and consequently we can write

$$
\delta\left(r_{0} ; \omega, \nu\right)=\sum_{j=2}^{2 k-1} v_{j}(\omega, \nu) r_{0}^{j}+v_{2 k}(\omega, \nu)\left[1+\psi\left(r_{0} ; \omega, \nu\right)\right] r_{0}^{2 k} .
$$

Now using standard arguments (see for example Proposition 6.1 .2 of [31]) we known that an upper bound on the cyclicity of the focus at the origin with respect to perturbation within the family (3) is given by $2(k-1)$. Actually, this bound can be improved sometimes using a special basis of the ideal $\hat{\mathcal{I}}_{k}$ which we simply call "minimal basis" but is, in fact, a minimal basis with respect to the ordered integrability focus quantities.

Definition 5. Consider the ideal $\hat{\mathcal{I}}_{k}$ in the Noetherian ring $\mathbb{R}(\omega)[\nu]$. For some $s \leq k$ we say that the basis $B=\left\{\hat{\eta}_{j_{1}}, \ldots, \hat{\eta}_{j_{s}}\right\}$ of $\hat{\mathcal{I}}_{k}$ is minimal if it satisfies the following properties:

(i) $\hat{\eta}_{i} \equiv 0$ for $1 \leq i \leq j_{1}-1$ and $\hat{\eta}_{j_{1}} \not \equiv 0$;

(ii) For $j_{1}+1 \leq i \leq k, \hat{\eta}_{i} \in B$ if and only if $\hat{\eta}_{i} \notin \hat{\mathcal{I}}_{i-1}$.

Of course similar definitions apply to obtain minimal basis of $\mathcal{B}_{2 k}, \mathcal{B}$ or $\hat{\mathcal{I}}$.

Let $k$ be the order of the focus at the origin of (3) with $(\omega, \nu)=\left(\omega^{\dagger}, \nu^{\dagger}\right)$ so that, according to Theorem $2, \hat{\eta}_{j}\left(\omega^{\dagger}, \nu^{\dagger}\right)=0$ for $j \leqslant k-1$ and $\hat{\eta}_{k}\left(\omega^{\dagger}, \nu^{\dagger}\right) \neq 0$. 
Let $\left\{\hat{\eta}_{j_{1}}, \ldots, \hat{\eta}_{j_{s}}\right\}$ be a minimal basis of the ideal $\hat{\mathcal{I}}_{k}$ having $s$ elements and where $j_{s}=k$. Then $s \leq k$ and near $\left(\omega^{\dagger}, \nu^{\dagger}\right)$ we can rearrange $\delta$ as

$$
\delta\left(r_{0} ; \omega, \nu\right)=\sum_{i=1}^{s-1} \hat{\eta}_{j_{i}}(\omega, \nu)\left[1+\hat{\psi}_{i}\left(r_{0} ; \omega, \nu\right)\right] r_{0}^{2 j_{i}+1}+\hat{\eta}_{k}(\omega, \nu)\left[1+\hat{\psi}\left(r_{0} ; \omega, \nu\right)\right] r_{0}^{2 k+1}
$$

producing the upper bound $s-1$ on the cyclicity of the focus.

On the contrary, now we assume that system (3) with $(\omega, \nu)=\left(\omega^{*}, \nu^{*}\right) \in \Omega \times \mathbb{R}^{p}$ has a center at the origin, that is $v_{j}\left(\omega^{*}, \nu^{*}\right)=0$ for all $j \in \mathbb{N}$. Let $m \in \mathbb{N}$ denote the cardinality of the minimal basis $\left\{v_{j_{1}}, \ldots, v_{j_{m}}\right\}$ of the Bautin ideal $\mathcal{B}$. This $m$ is called the the Bautin depth of $\mathcal{B}$. Then, for $(\omega, \nu)$ sufficiently close to $\left(\omega^{*}, \nu^{*}\right)$, the displacement map can be expressed as

$$
\delta\left(r_{0} ; \omega, \nu\right)=\sum_{k=1}^{m} v_{j_{k}}(\omega, \nu)\left[1+\psi_{k}\left(r_{0} ; \omega, \nu\right)\right] r_{0}^{j_{k}}
$$

where $\psi_{k}\left(r_{0} ; \omega, \nu\right)$ are analytic functions at $r_{0}=0$. The reader can see a proof of this rearrangement in Lemma 6.1 .6 of [31]. From (7) and by a repeated use Rolle's Theorem the following result follows.

Theorem 6. Assume the origin is a center of system (3) with $(\omega, \nu)=\left(\omega^{*}, \nu^{*}\right) \in$ $\Omega \times \mathbb{R}^{p}$. Let $m \in \mathbb{N}$ be the Bautin depth of $\mathcal{B}=\left\langle v_{j}(\omega, \nu): j \in \mathbb{N}\right\rangle$ in the ring $\mathbb{R}(\omega)[\nu]$. Then the cyclicity of the center with respect to perturbations within the family (3) is at most $m-1$.

Although valuable from a theoretical point of view, in general it is very difficult to compute the Bautin depth of $\mathcal{B}$ in practical cases, so we need to develop other tools besides Theorem 6 to bound the center cyclicity of (3). This is the goal of the next section.

2.2. The ideas of [20] adapted to the nilpotent singularity. Following exactly the same ideas than in [20] we can obtain a center cyclicity bound computed with polynomial ideals. The proofs are essentially the same (therefore we do not reproduce them here) if we replace the concepts Hopf singularity in $\mathbb{R}^{3}$, formal first integral, focal values and admissible parameter $\lambda \in \mathbb{R}^{*}$ by nilpotent singularity with Andreev number $(n, \hat{\beta})=(2,1)$, formal inverse integrating factor, integrating focal values and admissible parameter $\omega \in \Omega$, respectively.

The precise statement requires first some definitions. Since for a polynomial family (3) both $v_{j}$ and $\hat{\eta}_{j}$ are in $\mathbb{R}(\omega)[\nu]$, they can be expressed like

$$
v_{j}(\omega, \nu)=\frac{V_{j}(\omega, \nu)}{D_{j}(\omega)}, \quad V_{j} \in \mathbb{R}[\omega, \nu], \quad D_{j} \in \mathbb{R}[\omega]
$$

and where the roots of $D_{j}$ are not in $\Omega$. Similarly,

$$
\hat{\eta}_{j}(\omega, \nu)=\frac{g_{j}(\omega, \nu)}{d_{j}(\omega)}, \quad g_{j} \in \mathbb{R}[\omega, \nu], \quad d_{j} \in \mathbb{R}[\omega], \quad d_{j}^{-1}(0) \notin \Omega .
$$

Definition 7. We define the polynomial ideals $\mathcal{G}=\left\langle g_{j}: j \in \mathbb{N}\right\rangle, \mathcal{G}_{k}=\left\langle g_{1}, g_{2}, \ldots, g_{k}\right\rangle$, $\mathcal{V}=\left\langle V_{j}: j \in \mathbb{N}\right\rangle$ and $\mathcal{V}_{k}=\left\langle V_{2}, V_{3}, \ldots, V_{k}\right\rangle$ in the ring $\mathbb{R}[\omega, \nu]$.

It was proved in [20] that if $\left\{V_{j_{1}}, \ldots, V_{j_{m}}\right\}$ is the minimal basis of the polynomial ideal $\mathcal{V}$ with respect to the ordered basis $\left\{V_{j}: j \geqslant 2\right\}$ then $\left\{v_{j_{1}}, \ldots, v_{j_{m}}\right\}$ is a basis (not necessarily minimal) of the Bautin ideal $\mathcal{B}$ that satisfies the retention condition with respect to the ordered basis $\left\{v_{j}: j \geqslant 2\right\}$. Therefore the Bautin depth of the 
Bautin ideal $\mathcal{B}$ is at most $m$. Combining this fact together with Theorem 6 produces the following bound for the center cyclicity in terms of polynomial ideals.

Theorem 8. Let $m$ be the cardinality of the minimal basis of the polynomial ideal $\mathcal{V}=\left\langle V_{j}: j \in \mathbb{N}\right\rangle$ (equivalently, $\mathcal{G}=\left\langle g_{j}: j \in \mathbb{N}\right\rangle$ ) associated to a polynomial family (3). Then for any system (3) corresponding to parameter values $(\omega, \nu) \in \mathbf{V}_{\mathbb{R}}(\mathcal{V})$ with $\omega \in \Omega$, the cyclicity of the center at the origin, with respect to perturbation within the family (3), is at most $m-1$.

Theorem 8 is theoretically interesting but hard to use in concrete applications. The key point is how to compute the cardinality of the minimal basis of $\mathcal{V}$ or $\mathcal{G}$. As we will see later, to do that it is necessary to move over the complexes in order to use the completeness of $\mathbb{C}$ and Hilbert Nullstellensatz. Hence, we allow $(\omega, \nu) \in \mathbb{C} \times \mathbb{C}^{p}$ and we regard $\mathcal{G}=\left\langle g_{j}: j \in \mathbb{N}\right\rangle$ as an ideal in the complex polynomial ring $\mathbb{C}[\omega, \nu]$, see the forthcoming Theorem 14. However, after this complexification some complications arise such as we lose the geometrical ideas (for example symmetries) for identifying existence of a formal inverse integrating factor. For our purposes the most important step consists on checking that the equality of real varieties $\mathbf{V}_{\mathbb{R}}(\mathcal{G})=\mathbf{V}_{\mathbb{R}}\left(\mathcal{G}_{k}\right)$ for some $k \in \mathbb{N}$ implies the equality of complex varieties $\mathbf{V}_{\mathbb{C}}(\mathcal{G})=\mathbf{V}_{\mathbb{C}}\left(\mathcal{G}_{k}\right)$. The analysis of the former implication gets complicated mainly because when $\omega \in \mathbb{C} \backslash \Omega$ then additional parameter restrictions may appear in order to obtain a formal inverse integrating factor. The outcome is that the variety $\mathbf{V}_{\mathbb{C}}(\mathcal{G})$ need not exactly pick out systems with a formal inverse integrating factor. Actually, when $\omega \in \mathbb{C} \backslash \Omega$ it may happen that complex families with a formal inverse integrating factor are proper subsets of $\mathbf{V}_{\mathbb{C}}(\mathcal{G})$. In summary, we do not have a characterization in terms of formal inverse integrating factors of the variety $\mathbf{V}_{\mathbb{C}}(\mathcal{G})$ because there are points $(\omega, \nu)$ on it with $\omega \in \mathbb{C} \backslash \Omega$ for which the associated complex system (3) has no formal inverse integrating factor. The next example just illustrates what we have said.

Proposition 9. The polynomial family

$$
\dot{x}=-y+y\left(A x+B x^{2}\right), \quad \dot{y}=x^{3}+\omega x y
$$

in the form (3) with parameters $\omega$ and $\nu=(A, B)$ satisfy the following properties:

(i) If $(\omega, \nu) \in \mathcal{E}=\Omega \times \mathbb{R}^{2}$ then (10) possesses a formal inverse integrating factor in $\mathbb{R}[[x, y]]$ if and only if $A=0$.

(ii) If $\omega=0$ then $V(x, y)=-1+x(A+B x) \in \mathbb{C}[[x, y]]$ is an inverse integrating factor of (10) in $\mathbb{C}^{2}$.

Proof. When $(\omega, \nu) \in \mathcal{E}$ we find the first integrating focus quantity $\eta_{1}=-2 A \omega / 15$. Therefore $A=0$ is a necessary center condition which turns out to be sufficient because (10) is time reversible with respect to the symmetry $(x, y, t) \mapsto(-x, y,-t)$. This proves (i) since centers are characterized by existence of a formal inverse integrating factor in family (10). Statement (ii) is just straightforward.

Now we present a technical proposition useful in applications of the forthcoming Theorem 14 when the allowed perturbations in family (3) keep the value of $\omega$ constant. Before, we need the following definition.

Definition 10. Given a field $\mathbb{K}$ and an ideal $I=\left\langle f_{1}(\omega, \nu), \ldots, f_{k}(\omega, \nu)\right\rangle$ in $\mathbb{K}[\omega, \nu]$ let $I^{*}$ denote the ideal in $\mathbb{K}[\nu]$ that is generated by the polynomials $f_{j}^{*}(\nu)=f_{j}\left(\omega^{*}, \nu\right)$ for a fixed value $\omega=\omega^{*} \in \mathbb{K}$. 
The next proposition is the adaptation of Proposition 29 in [20] to our setting.

Proposition 11. Suppose $\mathbf{V}_{\mathbb{R}}\left(\mathcal{G}_{k}\right)=\mathbf{V}_{\mathbb{R}}(\mathcal{G}) \subset \mathbb{R}^{p+1}$ and $\left\{f_{1}, \ldots, f_{r}\right\} \subset \mathbb{R}[\omega, \nu]$ are such that

(a) $r \leqslant p+1$,

(b) $\mathbf{V}_{\mathbb{C}}\left(\mathcal{G}_{k}\right)=\mathbf{V}_{\mathbb{C}}\left(f_{1}, \ldots, f_{r}\right)$, and

(c) for some $\Sigma \varsubsetneqq \mathbf{V}_{\mathbb{C}}\left(\mathcal{G}_{k}\right)$ the map $F: \mathbb{C}^{p+1} \rightarrow \mathbb{C}^{r}:(\omega, \nu) \mapsto\left(f_{1}(\omega, \nu), \ldots, f_{r}(\omega, \nu)\right)$ satisfies $\operatorname{rank}\left(d_{P} F\right)=r$ at every point $P \in \mathbf{V}_{\mathbb{C}}\left(\mathcal{G}_{k}\right) \backslash \Sigma$.

Then, if the Zariski closure $\overline{\mathbf{V}_{\mathbb{C}}\left(\mathcal{G}_{k}\right) \backslash \Sigma}=\mathbf{V}_{\mathbb{C}}\left(\mathcal{G}_{k}\right)$ then $\mathbf{V}_{\mathbb{C}}\left(\mathcal{G}_{k}^{*}\right)=\mathbf{V}_{\mathbb{C}}\left(\mathcal{G}^{*}\right)$ in $\mathbb{C}^{p}$ when $\omega$ is assigned to any fixed value $\omega^{*} \in \Omega$.

Remark 12. Although implicit in the proofs of [20] we want here to point out that Proposition 11 remains true if we substitute the condition $\mathbf{V}_{\mathbb{R}}\left(\mathcal{G}_{k}\right)=\mathbf{V}_{\mathbb{R}}(\mathcal{G})$ by the weaker assumption $\mathbf{V}_{\mathbb{R}}\left(\mathcal{G}_{k}\right) \cap \mathcal{E}=\mathbf{V}_{\mathbb{R}}(\mathcal{G}) \cap \mathcal{E}$, where $\mathcal{E}$ is the parameter space of (3). This refinement, although unimportant in the examples presented in [20] is crucial in some examples of this work.

Remark 13. In applications often the set $\Sigma$ in condition (c) of Proposition 11 satisfies $\Sigma \subset \cup_{j \neq \ell}\left(C_{j} \cap C_{\ell}\right)$ where the sets $C_{s}$ are the irreducible components of $\mathbf{V}_{\mathbb{C}}\left(\mathcal{G}_{k}\right)$. Since by definition the Zariski closure of a set is the smallest variety that contains that set, in such a case the condition $\overline{\mathbf{V}_{\mathbb{C}}\left(\mathcal{G}_{k}\right) \backslash \Sigma}=\mathbf{V}_{\mathbb{C}}\left(\mathcal{G}_{k}\right)$ is automatic.

The following theorem is the version of Theorem 26 in [20] adapted to the nilpotent monodromic singularity class $(n, \hat{\beta})=(2,1)$ which is actually based on some ideas of the papers [25] and [15].

Theorem 14. Let (3) be a complex polynomial family on $\mathbb{C}^{2}$ with parameters $(\omega, \nu) \in \mathbb{C} \times \mathbb{C}^{p}$. Assume the equality $\mathbf{V}_{\mathbb{C}}(\mathcal{G})=\mathbf{V}_{\mathbb{C}}\left(\mathcal{G}_{k}\right)$ holds and that the minimal basis of $\mathcal{G}_{k}$ has cardinality $m$.

(i) If $\mathcal{G}_{k}$ is radical $\left(\sqrt{\mathcal{G}_{k}}=\mathcal{G}_{k}\right)$ then $\mathcal{G}=\mathcal{G}_{k}$ and for any $\left(\omega^{*}, \nu^{*}\right) \in \mathbf{V}_{\mathbb{R}}(\mathcal{G})$ with $\omega^{*} \in \Omega$ the cyclicity of the center at the origin perturbing within the real family (3) on $\mathbb{R}^{2}$ is at most $m-1$.

(ii) Let $\mathcal{G}_{k}=\mathcal{R} \cap \mathcal{N}$ be a primary decomposition of $\mathcal{G}_{k}$ where $\mathcal{R}$ is the intersection of the ideals in the decomposition that are prime (hence is radical) and $\mathcal{N}$ is the intersection of the remaining ideals. Then for any system of the real family (3) on $\mathbb{R}^{2}$ corresponding to parameters $\left(\omega^{*}, \nu^{*}\right) \in \mathbf{V}_{\mathbb{R}}(\mathcal{G}) \backslash \mathbf{V}_{\mathbb{R}}(\mathcal{N})$ with $\omega^{*} \in \Omega$ the cyclicity of the center at the origin is at most $m-1$.

Another approach for the center cyclicity focusing on the irreducible components of the center variety is the following one. For any $\kappa \in \mathbb{N}$ less or equal to the Bautin depth of $\mathcal{B}$, we denote by $d_{P} G_{\kappa}$ the $\kappa \times(p+1)$ Jacobian matrix of the real analytic mapping

$$
(\omega, \nu) \mapsto G_{\kappa}(\omega, \nu)=\left(\hat{\eta}_{j_{1}}(\omega, \nu), \ldots, \hat{\eta}_{j_{\kappa}}(\omega, \nu)\right),
$$

evaluated at a point $P \in \Omega \times \mathbb{R}^{p} \subset \mathbb{R}^{p+1}$, where $\left\{\hat{\eta}_{j_{1}}(\omega, \nu), \ldots, \hat{\eta}_{j_{\kappa}}(\omega, \nu)\right\}$ is the minimal basis of the ideal $\hat{\mathcal{I}}_{j_{\kappa}}$. Then, adapting Theorem 32 in [20] to our setting we obtain the following result which is in fact based on results in [10].

Theorem 15. Let $C \subset \mathbf{V}_{\mathbb{R}}(\mathcal{G})$ be an irreducible component of the center variety associated to the origin of the polynomial family $(3)$. Let $P=\left(\omega^{*}, \nu^{*}\right) \in C \cap \mathcal{E}$ with parameter space $\mathcal{E}=\left\{(\omega, \nu) \in \Omega \times \mathbb{R}^{p}\right\}$ be a point such that $\operatorname{rank}\left(d_{P} G_{\kappa}\right)=\kappa$ and $\kappa \leqslant p+1$. Then the following holds: 
(i) There exists a neighborhood $U$ of $P$ in $\mathbb{R}^{p+1}$ such that $C \cap U$ is a submanifold of $\mathbb{R}^{p+1}$ of codimension at least $\kappa$ and there exist bifurcations of (3) producing $\kappa-1$ small amplitude limit cycles from the origin for parameter values with $(\omega, \nu)$ sufficiently close to $P$.

(ii) If moreover $\operatorname{codim}(C)=\kappa$ then $P$ is a smooth point of $C$ and the cyclicity of $P$ and also of any point in a relatively dense open subset of $C$ is exactly $\kappa-1$.

2.3. Examples. In this section we apply the above developed theory to some concrete families (3). In these examples an intensive use of several routines in the primdec.lib library [13] of Singular [12] is necessary. Thus, given a polynomial ideal $\mathcal{I}$, we can use the routine minAssChar to obtain the prime decomposition of $\sqrt{\mathcal{I}}$. We also can check whether a polynomial ideal $\mathcal{I}$ is radical or not thanks to the primdecGTZ or primdecSY routines.

Once we know the cyclicity bound of a focus due to its order or the center cyclicity thanks to the Bautin depth, the next natural step is trying to prove that the bound is indeed sharp. Then we need to make a concrete perturbation within the family that creates the maximum number of limit cycles. If it exists, the details on the choice of that perturbations are explained below.

Remark 16. Let $k$ be maximum order of a focus at the origin of (3) with parameter space $\mathcal{E}$. In other words, $k$ is the minimum integer such that the equality $\mathbf{V}_{\mathbb{R}}\left(\mathcal{G}_{k}\right) \cap$ $\mathcal{E}=\mathbf{V}_{\mathbb{R}}(\mathcal{G}) \cap \mathcal{E}$ holds. Assume the focus corresponds to the parameter values $(\omega, \nu)=\left(\omega^{\dagger}, \nu^{\dagger}\right)$ so that $\tilde{\eta}_{j}\left(\omega^{\dagger}, \nu^{\dagger}\right)=0$ for $j=1, \ldots, k-1$ but $\tilde{\eta}_{j}\left(\omega^{\dagger}, \nu^{\dagger}\right) \neq 0$. Let $\left\{\hat{\eta}_{j_{1}}(\gamma(\varepsilon)), \ldots, \hat{\eta}_{j_{s}}(\gamma(\varepsilon))\right\}$ with $j_{s}=k$ be a minimal basis of the ideal $\hat{\mathcal{I}}_{k}$ of cardinality $s \leq k$. Let us perturb along an analytic curve $\varepsilon \mapsto \gamma(\varepsilon)=(\omega(\varepsilon), \nu(\varepsilon)) \subset$ $\mathcal{E}$ with $\gamma(0)=\left(\omega^{\dagger}, \nu^{\dagger}\right)$ in such a way that, for $|\varepsilon| \ll 1$, the chain of inequalities

$$
\left|\hat{\eta}_{j_{1}}(\gamma(\varepsilon))\right| \ll\left|\hat{\eta}_{j_{2}}(\gamma(\varepsilon))\right| \ll \cdots \ll\left|\hat{\eta}_{j_{s}}(\gamma(\varepsilon))\right| \neq 0,
$$

holds with the additional change of consecutive signs $\hat{\eta}_{j_{i}}(\gamma(\varepsilon)) \hat{\eta}_{j_{i+1}}(\gamma(\varepsilon))<0$ for $i=1, \ldots, s-1$. Then by using standard arguments of bifurcation theory it follows that $s-1$ small amplitude limit cycles can be made to bifurcate from the origin of (3) by perturbing in $\mathcal{E}$ from $\left(\omega^{\dagger}, \nu^{\dagger}\right)$.

The limit cycle realization perturbing a center is basically equal. Assume that $\mathbf{V}_{\mathscr{C}} \cap \mathcal{E}=\mathbf{V}_{\mathbb{R}}\left(\mathcal{G}_{r}\right) \cap \mathcal{E}$ for certain index $r$ and let $\left\{\hat{\eta}_{k_{1}}(\gamma(\varepsilon)), \ldots, \hat{\eta}_{k_{m}}(\gamma(\varepsilon))\right\}$ with $k_{m}=r$ be a minimal basis of the ideal $\hat{\mathcal{I}}_{r}$ of cardinality $m \leq r$. Assume the origin is a center of $(3)$ when $(\omega, \nu)=\left(\omega^{*}, \nu^{*}\right)$ so that $\tilde{\eta}_{j}\left(\omega^{*}, \nu^{*}\right)=0$ for all $j \in \mathbb{N}$. If we perturb by $\varepsilon \mapsto \gamma(\varepsilon)=(\omega(\varepsilon), \nu(\varepsilon)) \subset \mathcal{E}$ with $\gamma(0)=\left(\omega^{*}, \nu^{*}\right)$ satisfying

$$
\left|\hat{\eta}_{k_{1}}(\gamma(\varepsilon))\right| \ll\left|\hat{\eta}_{k_{2}}(\gamma(\varepsilon))\right| \ll \cdots \ll\left|\hat{\eta}_{k_{m}}(\gamma(\varepsilon))\right| \ll 1,
$$

and $\hat{\eta}_{k_{i}}(\gamma(\varepsilon)) \hat{\eta}_{k_{i+1}}(\gamma(\varepsilon))<0$ for $i=1, \ldots, m-1$ then, for $|\varepsilon| \ll 1, m-1$ limit cycles bifurcate from the center at the origin of (3) by perturbing in $\mathcal{E}$ from $\left(\omega^{*}, \nu^{*}\right)$.

2.3.1. Example 1. In [1] the authors consider the following polynomial family

$$
\dot{x}=y+a x y+b y^{2}, \quad \dot{y}=-x^{3}+k x y+c y^{3} .
$$

Computing the functions $F, f$ and $\varphi$ of Theorem 22 for this system gives $F(x) \equiv 0$, $f(x)=-x^{3}$ and $\varphi(x)=k x$. Therefore the origin is a monodromic nilpotent singularity if and only if $8-k^{2}>0$. Moreover, is of type $(n, \hat{\beta})=(2,1)$ if and only if, additionally, $k \neq 0$. The center problem at the origin of the full family (12) was solved in [1]. Restricting their result to $k \neq 0$, it follows that the only center 
corresponds to the time-reversible case $a=c=0$. In the following theorem we analyze the cyclicity problem at the origin.

Theorem 17. The center variety $\mathbf{V}_{\mathscr{C}}$ associated to the origin of family (12) with parameter space $\mathcal{E}=\left\{(\omega, \nu)=(-k, a, b, c) \in \Omega \times \mathbb{R}^{3}\right\}$ is given by

$$
\mathbf{V}_{\mathscr{C}}=\left\{(\omega, \nu) \in \mathbb{R}^{4}: a=c=0\right\} .
$$

The origin is a center of (12) if and only if $(\omega, \nu) \in \mathbf{V}_{\mathscr{C}} \cap \mathcal{E}=\mathbf{V}_{\mathbb{R}}\left(\mathcal{G}_{2}\right) \cap \mathcal{E}$. Moreover the following holds:

(i) The cyclicity of any center at the origin under perturbations within family (12) is 1;

(ii) The cyclicity of any focus of (12) at the origin is bounded by 1 and there are foci with cyclicity 1.

2.3.2. Example 2. Consider the polynomial Andreev's canonical family (3) with $\tilde{P}$ and $\tilde{Q}$ general $(1,2)$-quasihomogeneous polynomials of minimal degrees, that is, degrees 1 and 4 respectively. This family is given by

$$
\dot{x}=-y+A x y, \quad \dot{y}=x^{3}+\omega x y+D x^{4}+E x^{2} y+F y^{2},
$$

with parameters $\omega \in \Omega$ and $\nu=(A, D, E, F) \in \mathbb{R}^{4}$.

Theorem 18. The center variety $\mathbf{V}_{\mathscr{C}}$ associated to the origin of family (14) has 7 irreducible components: $\mathbf{V}_{\mathscr{C}}=\cup_{j=1}^{7} C_{i} \subset \mathbb{R}^{5}$ where

$$
\begin{aligned}
& C_{1}=\left\{(\omega, \nu) \in \mathbb{R}^{5}: 6 D+F=2 A-3 F=\omega F+2 E=0\right\}, \\
& C_{2}=\left\{(\omega, \nu) \in \mathbb{R}^{5}: 3 D-2 F=A+F=\omega F-E=0\right\}, \\
& C_{3}=\left\{(\omega, \nu) \in \mathbb{R}^{5}: 2 A+2 D-F=\omega D-E=0\right\}, \\
& C_{4}=\left\{(\omega, \nu) \in \mathbb{R}^{5}: 3 D+2 F=4 A-3 F=\omega F+2 E=0\right\}, \\
& C_{5}=\left\{(\omega, \nu) \in \mathbb{R}^{5}: 3 D+2 F=3 A-F=\omega F+3 E=0\right\}, \\
& C_{6}=\left\{(\omega, \nu) \in \mathbb{R}^{5}: E=9 D+F=3 A-F=0\right\}, \\
& C_{7}=\left\{(\omega, \nu) \in \mathbb{R}^{5}: E=D+F=A+F=0\right\} .
\end{aligned}
$$

The origin is a center of (14) with parameter space $\mathcal{E}=\{(\omega, \nu)=(\omega, A, D, E, F) \in$ $\left.\Omega \times \mathbb{R}^{4}\right\}$ if and only if $(\omega, \nu) \in \mathbf{V}_{\mathscr{C}} \cap \mathcal{E}=\mathbf{V}_{\mathbb{R}}\left(\mathcal{G}_{4}\right) \cap \mathcal{E}$. Moreover the following holds:

(i) The cyclicity of any center at the origin of (14) having parameter values $(\omega, \nu) \in C_{j} \cap \mathcal{E}$ with $j \neq 3$ under perturbations within family (14) is exactly 2 except when $\nu=(0,0,0,0)$;

(ii) The cyclicity of any center at the origin of (14) having parameter values $(\omega, \nu) \in C_{3} \cap \mathcal{E}$ perturbed within family (14) is exactly 1 under the restriction $(A+3 D)(2 A+3 D) \neq 0$

(iii) The cyclicity of the center at the origin of (14) when $\nu=(0,0,0,0)$ perturbed within family (14) is zero provided $\alpha(\omega, \nu) \not \equiv 0$ where the expression of $\alpha$ is given in (57).

(iv) The cyclicity of any focus of (14) at the origin is bounded by 2 and there are foci with cyclicity 2.

2.3.3. Example 3. In Theorem 4.1 of [5] the center problem of the following polynomial Andreev's canonical family (3) was analyzed:

$$
\dot{x}=-y, \quad \dot{y}=x^{3}+\omega x y+A x^{2} y+B y^{2}+C x y^{2}+D y^{3},
$$


with parameters $\omega \in \Omega$ and $\nu=(A, B, C, D) \in \mathbb{R}^{4}$. There it is proved that the origin is a center if and only if $A=B=D=0$, which corresponds to the timereversible stratum with symmetry $(x, y, t) \mapsto(-x, y,-t)$ and, moreover, that there exist systems inside family (15) with 3 small amplitude limit cycles bifurcating from a nilpotent focus at the origin, see Remark 33 . We expand this study analyzing the cyclicity of the origin in (15).

Theorem 19. The center variety $\mathbf{V}_{\mathscr{C}}$ associated to the origin of family (15) with parameter space $\mathcal{E}=\left\{(\omega, \nu)=(\omega, A, B, C, D) \in \Omega \times \mathbb{R}^{4}\right\}$ is given by

$$
\mathbf{V}_{\mathscr{C}}=\left\{(\omega, \nu) \in \mathbb{R}^{5}: A=B=D=0\right\} \text {. }
$$

The origin is a center of $(15)$ if and only if $(\omega, \nu) \in \mathbf{V}_{\mathscr{C}} \cap \mathcal{E}=\mathbf{V}_{\mathbb{R}}\left(\mathcal{G}_{4}\right) \cap \mathcal{E}$. Moreover the following holds:

(i) If $C \neq 0$ then the cyclicity of any center at the origin under perturbations within family (15) is exactly 2 ;

(ii) If $C=0$ then there are perturbations within family (15) producing 1 limit cycle bifurcating from the center at the origin.

(iii) The cyclicity of any focus of (15) at the origin is bounded by 3 and there are foci with cyclicity 3.

2.3.4. Example 4. We will analyze Liénard polynomial families of arbitrary degree $d \geq 4$ with linear damping inside the Andreev's canonical form (3). This family has the form

$$
\dot{x}=-y, \quad \dot{y}=g(x ; \nu)+y f(x ; \omega)
$$

with polynomials

$$
f(x ; \omega)=\omega x, \quad g(x ; \nu)=x^{3}+\sum_{j=4}^{d} \nu_{j} x^{j}
$$

and parameters $\omega \in \Omega$ and $\nu=\left(\nu_{4}, \ldots, \nu_{d}\right) \in \mathbb{R}^{d-3}$.

Theorem 20. The center variety $\mathbf{V}_{\mathscr{C}}$ associated to the origin of family (17) with parameter space $\mathcal{E}=\left\{(\omega, \nu) \in \Omega \times \mathbb{R}^{d-3}\right\}$ is given by

$$
\mathbf{V}_{\mathscr{C}}=\left\{(\omega, \nu) \in \mathbb{R}^{d-2}: \nu_{2 j}=0: j=2, \ldots, m+1\right\},
$$

with $2(m+1)=d$ or $2(m+1)=d-1$ according to whether $d$ is even or odd, respectively. The origin is a center of (17) if and only if $(\omega, \nu) \in \mathbf{V}_{\mathscr{C}} \cap \mathcal{E}=$ $\mathbf{V}_{\mathbb{R}}\left(\mathcal{G}_{m}\right) \cap \mathcal{E}$. Moreover the following holds:

(i) The cyclicity of any center at the origin under perturbations within family (17) is exactly $m-1$;

(ii) The cyclicity of any focus of (17) at the origin is bounded by $m-1$ and there are foci with cyclicity $m-1$.

2.3.5. Example 5. We study family $(3)$ with $\tilde{Q}(x, y ; \nu) \equiv 0$ and $\tilde{P}(x, y ; \nu)$ an arbitrary polynomial of degree 2 , that is,

$$
\dot{x}=-y+y\left(\nu_{1} x+\nu_{2} y+\nu_{3} x^{2}+\nu_{4} x y+\nu_{5} y^{2}\right), \quad \dot{y}=x^{3}+\omega x y,
$$

with parameter space $\mathcal{E}=\left\{(\omega, \nu)=\left(\omega, \nu_{1}, \nu_{2}, \nu_{3}, \nu_{4}, \nu_{5}\right) \in \Omega \times \mathbb{R}^{5}\right\}$. 
Theorem 21. The center variety $\mathbf{V}_{\mathscr{C}}$ associated to the origin of family (20) with parameter space $\mathcal{E}=\left\{(\omega, \nu) \in \Omega \times \mathbb{R}^{5}\right\}$ has the symmetric irreducible component

$$
C^{\text {sym }}=\left\{(\omega, \nu) \in \mathbb{R}^{6}: \nu_{1}=\nu_{4}=0\right\} \subset \mathbf{V}_{\mathscr{C}} .
$$

Moreover, the cyclicity of any symmetric center at the origin with $\omega^{2} \neq 7$ under perturbations within family (20) is exactly 1.

Computing more integrability focus quantities than the necessary to prove Theorem 21 we check that $\hat{\eta}_{j}(\omega, \nu) \equiv 0$ for $j=3, \ldots, 8$, provided $\hat{\eta}_{1}(\omega, \nu)=\hat{\eta}_{2}(\omega, \nu) \equiv 0$ (equivalently $\left.\omega \nu_{1}=\left(\omega^{2}-7\right) \nu_{4} \equiv 0\right)$ and this supports the following conjecture.

Conjecture: Family (20) with $\omega^{2}-7=\nu_{1}=0$ has a center at the origin.

If this conjecture is true then the center problem for the origin of family (20) is completely solved being the center variety $\mathbf{V}_{\mathscr{C}}=C \cup C^{\mathrm{sym}}$ with $C=\left\{(\omega, \nu) \in \mathbb{R}^{6}\right.$ : $\left.\omega^{2}-7=\nu_{1}=0\right\}$.

\section{BACKGROUND AND PRELIMINARIES}

3.1. Andreev's characterization of monodromic nilpotent singularities. The following theorem of Andreev [6] characterizes analytic systems (1) for which the origin is monodromic.

Theorem 22. For an analytic system of the form (1) with an isolated singularity at the origin let $y=F(x)$ be the unique solution of $y+P(x, y)=0$ such that $F(0)=F^{\prime}(0)=0$ and let

$$
f(x)=Q(x, F(x)) \quad \text { and } \quad \varphi(x)=(\partial P / \partial x+\partial Q / \partial y)(x, F(x)) .
$$

Let $a \neq 0$ and $\alpha \geq 2$ be such that $f(x)=a x^{\alpha}+\cdots$.

When $\varphi$ is not identically zero let $b \neq 0$ and $\hat{\beta} \geq 1$ be such that $\varphi(x)=b x^{\hat{\beta}}+\cdots$. Then the origin of (1) is monodromic if and only if $\alpha=2 n-1$ is an odd integer, $a<0$, and one of the following conditions holds:

(i) $\varphi(x) \equiv 0$

(ii) $\hat{\beta} \geq n$

(iii) $\hat{\beta}=n-1$ and $b^{2}+4 a n<0$.

In this work we will focus on the former monodromic case (iii) with Andreev's number $n=2$. In the study of the monodromic nilpotent singularities it is useful and natural to introduce $(1, n)$-quasihomogeneous polynomials with $n$ the associated Andreev's number.

Definition 23. A polynomial $p_{k} \in \mathbb{R}[x, y]$ is a $(1, n)$-quasihomogeneous polynomials of weighted degree $k$ if $p_{k}\left(\lambda x, \lambda^{n} y\right)=\lambda^{k} p_{k}(x, y)$ for all $\lambda \in \mathbb{R}$. In consequence we get $p_{k}(x, y)=\sum_{i+n j=k} a_{i j} x^{i} y^{j}$ for certain coefficients $a_{i j} \in \mathbb{R}$. On the other hand, a vector field $\mathcal{X}_{j}=p_{j+1} \partial_{x}+q_{j+n} \partial_{y}$ is a $(1, n)$-quasihomogeneous polynomial vector field of weighted degree $j$ if its components $p_{j+1}$ and $q_{j+n}$ are $(1, n)-$ quasihomogeneous polynomials of weighted degrees $j+1$ and $j+n$, respectively. 
3.2. The Andreev's canonical form. We consider system (1) and we assume that the origin is a nilpotent monodromic singular point with $(n, \hat{\beta})=(2,1)$, that is, we consider case (iii) in Theorem 22. Then, considering the function $F$ and the value $a \in \mathbb{R}$ of Theorem 22, we perform first the analytic change of variables $(x, y) \mapsto(x, y-F(x))$ and next the rescaling $(x, y) \mapsto(\alpha x,-\alpha y)$ with $\alpha=$ $(-1 / a)^{-1 / 2}$ bringing system (1) to the Andreev canonical form (3).

3.3. Generalized polar coordinates and the Poincaré map. The generalized trigonometric functions (adapted here for the Andreev's number $n=2$ ) were introduced by Lyapunov in [29] as the unique solution $x(\theta)=\operatorname{Cs} \theta$ and $y(\theta)=\operatorname{Sn} \theta$ of the Cauchy problem $\frac{d x}{d \theta}=-y, \frac{d y}{d \theta}=x^{3}$ with initial condition $(x(0), y(0))=(1,0)$. We introduce the generalized polar blow-up $(x, y) \mapsto(r, \theta)$ defined by

$$
x=r \operatorname{Cs} \theta, \quad y=r^{2} \operatorname{Sn} \theta
$$

embedding a neighborhood of the origin into the cylinder $C=\left\{(r, \theta) \in \mathbb{R} \times \mathcal{S}^{1}\right\}$ with $|r|$ sufficiently small. The change (22) transforms the Andreev canonical form (3) into the following ordinary differential equation defined on $C$ :

$$
\frac{d r}{d \theta}=\mathcal{F}(r, \theta ; \omega, \nu)
$$

where $\mathcal{F}$ is an analytic function on $C$ with $\mathcal{F}(0, \theta ; \omega, \nu) \equiv 0$ for all $\theta \in \mathcal{S}^{1}=\mathbb{R} / T \mathbb{Z}$ where $T$ is the minimal period of both $\operatorname{Cs} \theta$ and $\operatorname{Sn} \theta$, see [29]. More specifically (see for example [4] or [19]) we have

$$
\begin{aligned}
& \dot{r}=\mathcal{R}(r, \theta ; \omega, \nu)=\operatorname{Cs} \theta \operatorname{Sn}^{2} \theta r^{2}+O\left(r^{3}\right), \\
& \dot{\theta}=\Theta(r, \theta ; \omega, \nu)=\Theta_{0}(\theta ; \omega) r+O\left(r^{2}\right),
\end{aligned}
$$

where $\Theta_{0}(\theta ; \omega)=1+\omega \operatorname{Cs}^{2} \theta \operatorname{Sn} \theta>0$ for any $\theta \in[0, T)$ due to the monodromic condition $\omega \in \Omega$. From here we see that (23) has the form $\frac{d r}{d \theta}=\frac{\mathcal{R}(r, \theta ; \omega, \nu)}{\Theta(r, \theta ; \omega, \nu)}:=$ $\mathcal{F}(r, \theta ; \omega, \nu)$.

Let $\Psi\left(\theta ; r_{0}, \omega, \nu\right)$ be the solution of $(23)$ with initial condition $\Psi\left(0 ; r_{0}, \omega, \nu\right)=$ $r_{0}$. Then we can define the Poincaré map $\Pi: \Sigma \subseteq \mathbb{R} \rightarrow \mathbb{R}$ as $\Pi\left(r_{0} ; \omega, \nu\right)=$ $\Psi\left(T ; r_{0}, \omega, \nu\right)$. Thus $\Pi$ becomes an analytic diffeomorphism and, from the results of [18] and [19], it follows that $\Pi$ admits the Taylor expansion

$$
\Pi\left(r_{0} ; \omega, \nu\right)=r_{0}+\sum_{j \geq 2} v_{j}(\omega, \nu) r_{0}^{j},
$$

where $v_{j}$ are the Poincaré-Lyapunov quantities. In order to exemplify the difficulties we encounter when we try to compute the Poincaré-Lyapunov quantities, we see that

$$
v_{2}(\omega, \nu)=\int_{0}^{T} \mathcal{F}_{2}(\theta ; \omega, \nu) \exp \left(\int_{0}^{\theta} \mathcal{F}_{1}(\sigma ; \omega) d \sigma\right) d \theta
$$

where $\mathcal{F}(r, \theta ; \omega, \nu)=\sum_{j \geq 1} \mathcal{F}_{j}(\theta ; \omega, \nu) r^{j}$, and $\mathcal{F}_{1}(\theta ; \omega)=\omega \operatorname{Cs} \theta \operatorname{Sn}^{2} \theta / \Theta_{0}(\theta ; \omega) \not \equiv 0$ although $\int_{0}^{T} \mathcal{F}_{1}(\theta ; \omega) d \theta=0$, see [4] for details.

In [21] it is proved that when (3) is a polynomial family parameterized by the set of admissible coefficients then the Poincaré-Lyapunov quantities $v_{k}$ are polynomials in the parameters if and only if $\omega$ is a fixed constant, not a parameter. More precisely, we have that $v_{k} \in \mathbb{R}(\omega)[\nu]$. 
Remark 24. System (3) with $(\omega, \nu)=\left(\omega^{*}, \nu^{*}\right)$ and $\omega^{*} \in \Omega$ has a center at the origin if and only if $v_{j}\left(\omega^{*}, \nu^{*}\right)=0$ for any $j \in \mathbb{N}$ so that $\Pi\left(r_{0} ; \omega^{*}, \nu^{*}\right)=r_{0}$. On the other side, when $(3)$ with $(\omega, \nu)=\left(\omega^{\dagger}, \nu^{\dagger}\right)$ and $\omega^{\dagger} \in \Omega$ possesses a focus at the origin, the first subindex $k$ with $v_{k}\left(\omega^{*}, \nu^{*}\right) \neq 0$ is an even number.

3.4. The Poincaré map and inverse integrating factors. An inverse integrating factor of (23) is a function $v: C \rightarrow \mathbb{R}$ of class $C^{1}(C)$, hence a $T$-periodic function in the variable $\theta$, which is non-locally null and satisfies the partial differential equation:

$$
\frac{\partial v}{\partial \theta}(r, \theta ; \omega, \nu)+\frac{\partial v}{\partial r}(r, \theta ; \omega, \nu) \mathcal{F}(r, \theta ; \omega, \nu)=\frac{\partial \mathcal{F}}{\partial r}(r, \theta ; \omega, \nu) v(r, \theta ; \omega, \nu) .
$$

If $W(x, y ; \omega, \nu)$ is an inverse integrating factor of the Andreev canonical form (3) defined in a neighborhood of the nilpotent monodromic singularity at the origin then it is easy to prove that an inverse integrating factor $v(r, \theta)$ of the corresponding equation (23) is

$$
v(r, \theta ; \omega, \nu)=\frac{W\left(r \operatorname{Cs} \theta, r^{2} \operatorname{Sn} \theta ; \omega, \nu\right)}{r^{2} \Theta(r, \theta ; \omega, \nu)} .
$$

In [18] it is also proved the following fundamental result:

$$
v\left(\Pi\left(r_{0} ; \omega, \nu\right), 0 ; \omega, \nu\right)=v\left(r_{0}, 0 ; \omega, \nu\right) \Pi^{\prime}\left(r_{0} ; \omega, \nu\right),
$$

where the prime denotes derivative with respect to $r_{0}$.

\section{Proofs}

4.1. Proof of Theorem 1. Before giving the proof of Theorem 1 we need Lemma 25. This lemma appears in [1] but we reprove it again with our notations for the sake of completeness.

Given a diffeomorphism $\Phi$ on $\mathcal{U} \subset \mathbb{R}^{2}$ with $\operatorname{det}(D \Phi) \mid \mathcal{U} \neq 0$, we can apply the change of variables $\mathbf{x} \mapsto \mathbf{y}$ defined by $\mathbf{x}=\Phi(\mathbf{y})$ to a $C^{1}$ vector field $\mathcal{X}$ and a scalar function $f$, both defined on $U$. The outcome is the vector field $\Phi^{*}(\mathcal{X})$ and the function $\Phi^{*}(f)$ defined via the pull-back $\Phi^{*}(\mathcal{X}):=(D \Phi)^{-1} \mathcal{X} \circ \Phi$ and the function $\Phi^{*}(f):=f \circ \Phi$.

Lemma 25. Let $\mathcal{X}$ be a $C^{1}$-vector field on an open set $\mathcal{U} \subset \mathbb{R}^{2}$. Assume that a $C^{1}$ function $V: \mathcal{U} \rightarrow \mathbb{R}$ satisfies the linear partial differential equation

$$
\mathcal{X}(V)-V \operatorname{div}(\mathcal{X})=f
$$

for some scalar function $f$ defined on $\mathcal{U}$. Let $\Phi$ and $\xi$ be a diffeomorphism and a scalar function on $\mathcal{U}$ such that the restrictions $\left.\operatorname{det}(D \Phi)\right|_{\mathcal{U}} \neq 0$ and $\left.\xi\right|_{\mathcal{U}} \neq 0$. We define the pull-back vector field $\mathcal{Y}=\Phi^{*}(\xi \mathcal{X})$, that is, $\mathcal{Y}$ and $\mathcal{X}$ are orbitally equivalent. Then, the function $W=\Phi^{*}(\xi) \Phi^{*}(V) / \operatorname{det}(D \Phi)$ satisfies the linear partial differential equation

$$
\mathcal{Y}(W)-W \operatorname{div}(\mathcal{Y})=g
$$

where $g=\left(\Phi^{*}(\xi)\right)^{2} \Phi^{*}(f) / \operatorname{det}(D \Phi)$.

Proof. If we define $\tilde{V}=\Phi^{*}(V):=V \circ \Phi$ and $\tilde{\mathcal{Y}}=\Phi^{*}(\mathcal{X})$, first we claim that

$$
\mathcal{X}(V)=\tilde{\mathcal{Y}}(\tilde{V}) .
$$


This equality follows by the the chain rule and the push-forward definition. In short, defining $F(x)$ and $\tilde{F}(y)$ as the components of $\mathcal{X}$ and $\tilde{\mathcal{Y}}$ respectively, and using the dot to denote an ordinary scalar product, one has

$$
\begin{aligned}
\tilde{\mathcal{Y}}(\tilde{V}) & =\nabla_{y}(\tilde{V}) \cdot \tilde{F}=\nabla_{y}(V(\Phi(y))) \cdot \tilde{F}=\nabla_{x}(V(\Phi(y))) D \Phi(y) \cdot \tilde{F} \\
& =\nabla_{x}(V(\Phi(y))) D \Phi(y) \cdot(D \Phi(y))^{-1} F(\Phi(y))=\nabla_{x}(V(x)) \cdot F(x)=\mathcal{X}(V)
\end{aligned}
$$

proving the claim.

Now we define $\tilde{J}(y)=\operatorname{det}(D \Phi(y))$, so that $W(y)=\tilde{\xi}(y) J^{-1}(y) \tilde{V}(y)$ where $\tilde{\xi}=\xi \circ \Phi$ and also $J(x)=\tilde{J}\left(\Phi^{-1}(x)\right)$. Then, calling $G(x)$ the components of $\mathcal{Y}$ we have

$$
\begin{aligned}
g(y) & =\mathcal{Y}(W)-W \operatorname{div}(\mathcal{Y})=\nabla_{y}(W) \cdot G-W \operatorname{div}(G) \\
& =\nabla_{y}\left(\tilde{\xi} \tilde{J}^{-1} \tilde{V}\right) \cdot G-\tilde{\xi} \tilde{J}^{-1} \tilde{V} \operatorname{div}(G) \\
& =\left(\tilde{\xi} \tilde{J}^{-1} \nabla_{y}(\tilde{V})+\tilde{J}^{-1} \tilde{V} \nabla_{y}(\tilde{\xi})-\tilde{\xi} \tilde{V} \tilde{J}^{-2} \nabla_{y}(\tilde{J})\right) \cdot G-\tilde{\xi} \tilde{J}^{-1} \tilde{V} \operatorname{div}(G) .
\end{aligned}
$$

Now we analyze each one of these terms changing from variables $y$ to $x$, using (29) and recalling the definition of $\mathcal{Y}$. We obtain

$$
\begin{aligned}
\tilde{\xi} \tilde{J}^{-1} \nabla_{y}(\tilde{V}) \cdot G & =\xi J^{-1} \nabla_{x}(V) \cdot(\xi F)=\xi^{2} J^{-1} \nabla_{x}(V) \cdot F \\
\tilde{J}^{-1} \tilde{V} \nabla_{y}(\tilde{\xi}) \cdot G & =J^{-1} V \nabla_{x}(\xi) \cdot(\xi F)=\xi J^{-1} V \nabla_{x}(\xi) \cdot F \\
\tilde{\xi} \tilde{V} \tilde{J}^{-2} \nabla_{y}(\tilde{J}) \cdot G & =\xi V J^{-2} \nabla_{x}(J) \cdot(\xi F)=\xi^{2} V J^{-2} \nabla_{x}(J) \cdot F .
\end{aligned}
$$

In the last term we will apply the well-known relation

$$
\operatorname{div}(F)=\operatorname{div}(\tilde{F})+\tilde{J}^{-1} \nabla_{y} \tilde{J} . \tilde{F}
$$

see a proof in the book [30]. Therefore

$$
\begin{aligned}
\tilde{\xi} \tilde{J}^{-1} \tilde{V} \operatorname{div}(G) & =\xi J^{-1} V\left(\operatorname{div}(\xi F)-\tilde{J}^{-1} \nabla_{y}(\tilde{J}) \cdot(\xi F)\right. \\
& =\xi J^{-1} V\left(\operatorname{div}(\xi F)-J^{-1} \xi \nabla_{x}(J) \cdot F\right) \\
& =\xi J^{-1} V\left(\nabla_{x}(\xi) \cdot F+\xi \operatorname{div}(F)-J^{-1} \xi \nabla_{x}(J) . F\right)
\end{aligned}
$$

Finally we obtain that

$$
g=\xi^{2} J^{-1}\left(\nabla_{x}(V) \cdot F-V \operatorname{div}(F)\right)=\xi^{2} J^{-1} f
$$

proving the result.

Proof of Theorem 1. We make the proof with the help of the results proved in [26]. As noted in [26], after a nonlinear change of variables $(x, y) \mapsto\left(\alpha x, \alpha\left(y+K x^{2}\right)\right)$ with convenient $\alpha, K \in \mathbb{R}$, any vector field $\mathcal{X}$ with a monodromic nilpotent singularity at the origin with minimum Andreev number $(n, \hat{\beta})=(2,1)$ is taken towards

$$
\dot{x}=y+\lambda x^{2}+\hat{P}(x, y ; \mu), \quad \dot{y}=-2 x^{3}+2 \lambda x y+\hat{Q}(x, y ; \mu)
$$

with parameter space $\left\{(\lambda, \mu) \in \mathbb{R}^{*} \times \mathbb{R}^{p}\right\}$, where $\hat{P}$ and $\hat{Q}$ only contain $(1,2)$ quasihomogeneous terms of degrees greater than 2 and 3, respectively. Moreover, in Theorem 2.8 of [27] it is proved that if $\mathcal{X}$ is the vector field associated to (31) with $\lambda \neq 0$ then there is a unique formal series $V(x, y)=x^{4}+y^{2}+\sum_{j \geq 5} V_{j}(x, y)$ where $V_{j}$ are $(1,2)$-quasihomogeneous polynomials of weighted degree $j$ such that

$$
\mathcal{X}(V)-V \operatorname{div}(\mathcal{X})=x^{4} \sum_{j \geq 1} \eta_{j}(\lambda, \mu) x^{2 j}
$$


Our proof is a consequence of Lemma 25 applied to the particular case in which $\mathcal{X}$ is the field associated to (31), the linear partial differential equation under consideration is (32), and the conjugate field $\hat{\mathcal{X}}$ is in the Andreev's canonical form (3). In that case, the rescaling $\xi \equiv 1$. Moreover, if we compute the functions that appears in Theorem 22 in the particular case of a polynomial family (31) we obtain that the analytic function $F$ depends polynomially on the parameters $(\lambda, \mu)$. More concretely the function $F$ and $f$ of Theorem 22 have the Taylor expansions

$$
F(x ; \lambda, \mu)=-\lambda x^{2}+\cdots, \quad f(x ; \lambda, \mu)=-2\left(1+\lambda^{2}\right) x^{3}+\cdots .
$$

In particular, according with $\S 3.2$, the conjugation $(x, y) \mapsto \Phi(x, y)$ with

$$
\Phi(x, y)=(\alpha(\lambda) x,-\alpha(\lambda)[y-F(x ; \lambda, \mu)])
$$

and $\alpha(\lambda)=\sqrt{2\left(1+\lambda^{2}\right)}$ brings $\mathcal{X}$ to $\hat{\mathcal{X}}$, that is, transforms the polynomial family (31) into the analytic family of Andreev canonical forms (3) where $\omega(\lambda)=4 \lambda / \alpha(\lambda)$. We emphasize that the parameters $(\omega, \nu)$ of $(3)$ depend on the parameters $(\lambda, \mu)$ of (31) polynomially in $\mu$ but not in $\lambda$.

Recall that the diffeomorphism $\Phi$ used in Lemma 25 is just the inverse of the $\Phi$ defined in (33), that is,

$$
\Phi^{-1}(x, y)=(x / \alpha(\lambda),-y / \alpha(\lambda)+F(x / \alpha(\lambda) ; \lambda, \mu)) .
$$

In consequence, the functions $f$ and $g$ in Lemma 25 have the form $f(x, y)=$ $x^{4} \sum_{j \geq 1} \eta_{j}(\lambda, \mu) x^{2 j}$ and $g=\Phi_{*}(f) / \operatorname{det}\left(D \Phi^{-1}\right)$ where $\operatorname{det}\left(D \Phi^{-1}\right)=-1 / \alpha^{2}$ and, in coordinates, has the expression

$$
g(x, y)=-\frac{1}{\alpha^{2}}(x / \alpha)^{4} \sum_{j \geq 1} \eta_{j}(\lambda, \mu)(x / \alpha)^{2 j}=x^{4} \sum_{j \geq 1} \tilde{\eta}_{j}(\lambda, \mu) x^{2 j} .
$$

with $\tilde{\eta}_{j}(\lambda, \mu)=-\alpha^{-2(j+3)}(\lambda) \eta_{j}(\lambda, \mu)=-\left[2\left(1+\lambda^{2}\right)\right]^{-3-j} \eta_{j}(\lambda, \mu)$.

Finally, calling $\hat{W}$ the function $W$ in Lemma 25 we have $\hat{W}=\Phi_{*}(V) / \operatorname{det}\left(D \Phi^{-1}\right)$ where $V$ is defined in (32), that is,

$$
\hat{W}(x, y)=-\frac{1}{\alpha^{2}} V\left(\Phi^{-1}(x, y)\right)=-\frac{1}{\alpha^{2}} \sum_{j \geq 4} V_{j}\left(\Phi^{-1}(x, y)\right)=\sum_{j \geq 4} \hat{W}_{j}(x, y) .
$$

Since $V_{4}(x, y)=x^{4}+y^{2}$ and $F(x ; \lambda, \mu)=-\lambda x^{2}+\cdots$, we have

$$
\hat{W}_{4}(x, y)=-\frac{1}{\alpha^{2}}\left[\left(\frac{x}{\alpha}\right)^{4}+\left(-\frac{y}{\alpha}-\lambda\left(\frac{x}{\alpha}\right)^{2}\right)^{2}\right] \text {. }
$$

It follows that the series $W$ in the statement of the theorem is obtained by rescaling as $W=\hat{W} / \beta(\lambda)$ with $\beta(\lambda)=-1 /\left(8\left(1+\lambda^{2}\right)\right)$ and consequently the integrability focus quantities $\hat{\eta}_{j}$ in the statement of the theorem are just $\hat{\eta}_{j}(\omega(\lambda), \nu(\lambda, \mu))=$ $\tilde{\eta}_{j}(\lambda, \mu) / \beta(\lambda)=2^{-j}\left(1+\lambda^{2}\right)^{-j-1} \eta_{j}(\lambda, \mu)$ finishing the proof.

Remark 26. Of course, imitating the proof of Theorem 1, we are able to define integrability focus quantities $\eta_{j}^{*}$ associated to family (2) and relate them to integrability focus quantities $\eta_{j}$ for family (31). More concretely, $\mathcal{Y}(\bar{W})-\bar{W} \operatorname{div}(\mathcal{Y})=$ $x^{4} \sum_{k \geq 1} \eta_{k}^{*}(\Lambda) x^{2 k}$ where $\mathcal{Y}$ is the vector field associated to (2), the formal series $\bar{W}(x, y)=\sum_{k \geq 4} \bar{W}_{k}(x, y)$ where $\bar{W}_{k}$ are certain $(1,2)$-quasihomogeneous polynomials of weighted degree $k$ with $\bar{W}_{4}(x, y)=b x^{4}+(c-2 a) x^{2} y-2 y^{2}$ and where $\Lambda=(a, b, c, \zeta)$ is the vector parameter of $(2)$. Moreover $\eta_{j}^{*}=0$ if and only if $\eta_{j}=0$. 
Remark 27. As a particular case of the results of [32] it is known that (3) is orbitally analytically equivalent to a Liénard system. Let us take a polynomial such family

$$
\dot{x}=-y, \quad \dot{y}=x^{3}+y b(x ; \omega, \nu),
$$

with $b(x)=\omega x+\sum_{j=2}^{m} \nu_{j} x^{j}$ and $\omega \in \Omega$. Let $\mathcal{X}$ be the vector field associated to (34). Then, using the perspective of Theorem 1 and its proof we claim that there is a unique formal power series $V(x, y)=\sum_{k>4} V_{k}(x, y)$ where $V_{k}$ are certain $(1,2)$ quasihomogeneous polynomials of weighted degree $k$ and $V_{4}(x, y)=x^{4}+2 y^{2}+\omega x^{2} y$ such that

$$
\mathcal{X}(V)-V \operatorname{div}(\mathcal{X})=x^{4} \sum_{k \geq 1} \eta_{2 k}(\omega, \nu) x^{2 k} .
$$

Moreover, $\eta_{2 k}\left(\omega^{*}, \nu^{*}\right)=0$ for all $k \in \mathbb{N}$ if and only $b\left(x ; \omega^{*}, \nu^{*}\right)$ is an odd function, hence if and only if the origin is a center. A different proof of this claim consists on direct considerations using algebraic methods as we explain below.

Let $\mathcal{P}_{k}^{(1,2)} \subset \mathbb{R}[x, y]$ be the subspace of $(1,2)$-quasihomogeneous polynomials of weighted degree $k$, that is $p_{k}(x, y) \in \mathcal{P}_{k}^{(1,2)}$ if $p_{k}(x, y)=\sum_{i+2 j=k} a_{i j} x^{i} y^{j}$ with coefficients $a_{i j} \in \mathbb{R}$. A vector field $\mathcal{X}_{i}=p_{i+1} \partial_{x}+q_{i+2} \partial_{y}$ is said to be $(1,2)-$ quasihomogeneous polynomial vector field of weighted degree $i$ when $p_{i+1} \in \mathcal{P}_{i+1}^{(1,2)}$ and $q_{i+2} \in \mathcal{P}_{i+2}^{(1,2)}$.

Let $\mathcal{X}=-y \partial_{x}+\left(x^{3}+y b(x ; \omega, \nu) \partial_{y}\right.$ be the associated vector field to system (34). Then $\mathcal{X}=\sum_{i \geq 1} \mathcal{X}_{i}$ where $\mathcal{X}_{i}$ denotes a $(1,2)$-quasihomogeneous polynomial vector field of weighted degree $i$. More specifically we have $\mathcal{X}_{1}=-y \partial_{x}+\left(x^{3}+\omega x y\right) \partial_{y}$ and $\mathcal{X}_{i}=\nu_{i} x^{i} y \partial_{y}$ for any integer $i \geq 2$.

Notice that $\left\{x^{k}, x^{k-2} y, x^{k-4} y^{2}, \ldots, y^{k / 2}\right\}$ or $\left\{x^{k}, x^{k-2} y, x^{k-4} y^{2}, \ldots, x y^{(k-1) / 2}\right\}$ are the canonical basis of $\mathcal{P}_{k}^{(1,2)}$ when $k$ is even or odd, respectively. Therefore $\operatorname{dim} \mathcal{P}_{k}^{(1,2)}=\frac{k}{2}+1$ or $\operatorname{dim} \mathcal{P}_{k}^{(1,2)}=\frac{k-1}{2}+1$ depending on whether $k$ is even or odd, respectively. In particular, $\operatorname{dim} \mathcal{P}_{2 j}^{(1,2)}=\operatorname{dim} \mathcal{P}_{2 j+1}^{(1,2)}$ for any $j \in \mathbb{N}$.

We define the linear operator $\mathcal{L}: \mathcal{P}_{k}^{(1,2)} \rightarrow \mathcal{P}_{k+1}^{(1,2)}$ by $\mathcal{L}\left(P_{k}\right)=\mathcal{X}_{1}\left(P_{k}\right)-P_{k} \operatorname{div} \mathcal{X}_{1}$ where $\operatorname{div} \mathcal{X}_{1}=\omega x$. We have $\operatorname{dim} \mathcal{P}_{k}^{(1,2)}=\operatorname{dim}(\operatorname{ker} \mathcal{L})+\operatorname{dim}($ range $\mathcal{L})$. Notice that, when $k=4$ the kernel of $\mathcal{L}$ is one dimensional because ker $\mathcal{L}$ is generated by $V_{4}(x, y)=x^{4}+2 y^{2}+\omega x^{2} y$. On the contrary, if $k \geq 5$ then $\operatorname{ker} \mathcal{L}=\{0\}$ is the trivial one since $\mathcal{X}_{1}$ has no analytic first integral because $\omega \neq 0$.

Let us consider the left hand side $\mathcal{X}(V)-V \operatorname{div}(\mathcal{X})$ of (35). Taking into account that $\operatorname{div}(\mathcal{X})=b(x ; \omega, \nu)$ and the $(1,2)$-quasihomogeneous expansions $\mathcal{X}=\sum_{i \geq 1} \mathcal{X}_{i}$ and $V(x, y)=\sum_{k \geq 4} V_{k}(x, y)$ where $V_{k} \in \mathcal{P}_{k}^{(1,2)}$, we have

$$
\mathcal{X}(V)-V \operatorname{div}(\mathcal{X})=\left(\sum_{i \geq 1} \mathcal{X}_{i}\right)\left(\sum_{k \geq 4} V_{k}(x, y)\right)-\left(\sum_{k \geq 4} V_{k}(x, y)\right)\left(\omega x+\sum_{j=2}^{m} \nu_{j} x^{j}\right)
$$

Recalling that $\mathcal{L}\left(V_{4}\right) \equiv 0$, the terms in $\mathcal{P}_{k+1}^{(1,2)}$ with $k \geq 5$ of expression (36) are $\mathcal{L}\left(V_{k}\right)-F_{k+1}$, where the $F_{k+1} \in \mathcal{P}_{k+1}^{(1,2)}$ are $F_{k+1}(x, y)=\sum_{i+j=k+1} \nu_{i} x^{i} V_{j}(x, y)$ with $i \geq 2$ or, in other words, $F_{k+1}(x, y)=\sum_{i=2}^{k-3} \nu_{i} x^{i} V_{k+1-i}(x, y)$. Therefore (36) 
is written as

$$
\mathcal{X}(V)-V \operatorname{div}(\mathcal{X})=\sum_{k \geq 5}\left(\mathcal{L}\left(V_{k}\right)-F_{k+1}\right)
$$

Now we will prove the following claims.

(I) When $k$ is even, an arbitrary element $V_{k} \in \mathcal{P}_{k}^{(1,2)}$ has $\operatorname{dim} \mathcal{P}_{k}^{(1,2)}$ arbitrary coefficients which can be selected (solving linear algebraic equation) in such a way that the same number $\operatorname{dim} \mathcal{P}_{k}^{(1,2)}$ of coefficients of $\mathcal{L}\left(V_{k}\right)$ can be settled to have arbitrary values. In that case we will determine the unique coefficients of $V_{k}$ such that $\mathcal{L}\left(V_{k}\right)=F_{k+1}$.

(II) On the contrary, when $k$ is odd, the $\operatorname{dim} \mathcal{P}_{k}^{(1,2)}$ coefficients of an arbitrary polynomial $V_{k} \in \mathcal{P}_{k}^{(1,2)}$ can be chosen (solving again linear equation) in order that the $\operatorname{dim} \mathcal{P}_{k}^{(1,2)}+1$ coefficients of $\mathcal{L}\left(V_{k}\right)$ can be settled to belong to an arbitrary set of real numbers except one of them. This exceptional coefficient is just the coefficient of $x^{k+1}$. Thus, for $k$ odd, we can take with uniqueness the coefficients of $V_{k}$ in such a way that $\mathcal{L}\left(V_{k}\right)-F_{k+1}=$ $\eta_{k-3} x^{k+1}$ for certain integrating focal quantity $\eta_{k-3}$.

To prove the claims we will use induction over $k$. Denote by $A_{k}$ the matrix representation of the linear operator $\mathcal{L}: \mathcal{P}_{k}^{(1,2)} \rightarrow \mathcal{P}_{k+1}^{(1,2)}$ in the canonical basis. When $k$ is even $A_{k}$ is a square matrix of order $k / 2+1$ and when $k$ is odd the size of $A_{k}$ is $(k-1) / 2+2$ rows and $(k-1) / 2+1$ columns. It is easy to check by mathematical induction that $A_{k}$ is a tridiagonal matrix, that is a band matrix that has nonzero elements only on the main diagonal (those entries located at equal row and column), the first diagonal below this, and the first diagonal above the main diagonal. More specifically we have, for $k$ odd,

$$
A_{k}=\left(\begin{array}{cccccc}
-\omega & -1 & & & & \\
k & 0 & -2 & & & \\
& k-2 & \omega & -3 & & \\
& & k-4 & 2 \omega & \ddots & \\
& & & k-6 & \ddots & -\frac{k-1}{2} \\
& & & & \ddots & \left(\frac{k-1}{2}-1\right) \omega \\
& & & & & 1
\end{array}\right),
$$

and, when $k$ is even,

$$
A_{k}=\left(\begin{array}{ccccccc}
-\omega & -1 & & & & & \\
k & 0 & -2 & & & & \\
& k-2 & \omega & -3 & & & \\
& & k-4 & 2 \omega & \ddots & & \\
& & & k-6 & \ddots & \ddots & \\
& & & & \ddots & \ddots & -\frac{k}{2} \\
& & & & & 2 & \left(\frac{k}{2}-1\right) \omega
\end{array}\right) .
$$

First, we prove claim (II). To this end, we define the square matrix $A_{k}^{*}$ as the submatrix obtained after deleting the first row of matrix $A_{k}$ with $k$ odd. It follows that $A_{k}^{*}$ is upper triangular and $\operatorname{det} A_{k}^{*}=k ! !=k(k-2)(k-4) \cdots 1 \neq 0$, 
hence $A_{k}^{*}$ is nonsingular. This property of $A_{k}^{*}$ implies, in particular, claim (II). The reason is that the involved linear system to determine the coefficients $c_{i j}$ of $V_{k}(x, y)=\sum_{i+2 j=k} c_{i j} x^{i} y^{j}$ such that $\mathcal{L}\left(V_{k}\right)=F_{k+1}$ has associated matrix $A_{k}$. Therefore that linear system is overdetermined and, in general, has no solution. But, the linear system that satisfy the coefficients of $V_{k}$ and the $\eta_{k-3}$ such that $\mathcal{L}\left(V_{k}\right)=F_{k+1}+\eta_{k-3} x^{k+1}$ can be uniquely solved. This is because the subsystem obtained after deleting the first equation of that linear system has associated matrix $A_{k}^{*}$, hence it can be solved for all the coefficients of $V_{k}$ and, finally, we determine $\eta_{k-3}$ from the first equation.

To prove claim (I) first we recall a result on determinants of tridiagonal matrices. Let $B=\left(b_{i, j}\right)$ be a square tridiagonal matrix of order $n$ and denote $\Delta_{m}$ (with $m=1, \ldots, n)$ the principal minors, i.e., the determinants of the submatrices formed by the first $m$ rows and columns of $B$. From linear algebra, it is known that the determinant $\operatorname{det} B=\Delta_{n}$ can be computed from a three-term linear recurrence relation as follows: $\Delta_{m}=b_{m, m} \Delta_{m-1}-b_{m, m-1} b_{m-1, m} \Delta_{m-2}$ for all $3 \leq m \leq n$. In our case, applying this recurrence to the square tridiagonal matrix $A_{k}$ with $k$ even, we get

$$
\Delta_{m}(\omega)=(m-2) \omega \Delta_{m-1}(\omega)+(m-1)[k-2(m-2)] \Delta_{m-2}(\omega),
$$

for $3 \leq m \leq k / 2+1$ with initial conditions $\Delta_{1}=-\omega$ and $\Delta_{2}=k$. Clearly $\operatorname{det} A_{k}=\Delta_{k / 2+1}$ and claim (II) follows after proving that $\operatorname{det} A_{k} \neq 0$ for any $k \geq 6$ even under the constraint $\omega \in \Omega$.

4.2. Proof of Theorem 2. Before proving Theorem 2 we need the following preliminary result. Using the change of variables $\Phi$ on $\mathbb{R}^{2} \backslash\{(0,0)\}$ defined by (22) that transforms $(x, y) \mapsto(r, \theta)$ with Jacobian $r^{2}$ and next the time-rescaling $\xi=1 / \Theta(r, \theta)$ it follows that the vector field $\mathcal{X}$ associated to the Andreev canonical form (3) and the vector field $\overline{\mathcal{X}}=\partial_{\theta}+\mathcal{F}(r, \theta) \partial_{r}$ with $\mathcal{F}$ defined at (23) are orbitally equivalent on a sufficiently small punctured neighborhood of the origin. Then, using Lemma 25 we can prove the following result.

Corollary 28. Let $\overline{\mathcal{X}}=\partial_{\theta}+\mathcal{F}(r, \theta) \partial_{r}$ be the vector field defined by $(23)$ and $v(r, \theta)$ given by (27) where $W$ is defined in Theorem 1. Then

$$
\overline{\mathcal{X}}(v)-v \operatorname{div}(\overline{\mathcal{X}})=\frac{1}{r^{2} \Theta^{2}(r, \theta)} f(r \operatorname{Cs} \theta)
$$

where $f(x)=x^{4} \sum_{k \geq 1} \hat{\eta}_{k}(\omega, \nu) x^{2 k}$ and $\Theta$ is defined in (24).

Proof of Theorem 2. Let $r_{0}>0$ and $\Delta r_{0}>0$ both sufficiently small and $\Psi\left(\theta ; r_{0}\right)$ the solution of equation (23) with initial condition $\Psi\left(0 ; r_{0}\right)=r_{0}$. We take a simply connected domain $D$ in the coordinate plane $(\theta, r)$ whose boundary $\partial D$ is the union of the curves $\Gamma_{r_{0}}=\left\{\left(\theta, \Psi\left(\theta ; r_{0}\right)\right): 0 \leq \theta \leq T\right\}, \Gamma_{r_{0}+\Delta r_{0}}=\left\{\left(\theta, \Psi\left(\theta ; r_{0}+\Delta r_{0}\right)\right)\right.$ : $0 \leq \theta \leq T\}$, and the vertical segments $\ell_{0}=\left\{(0, r): r_{0} \leq r \leq r_{0}+\Delta r_{0}\right\}$ and $\ell_{T}=\left\{(T, r): \Pi\left(r_{0}\right) \leq r \leq \Pi\left(r_{0}+\Delta r_{0}\right)\right\}$.

We consider the vector field $\overline{\mathcal{X}}=\partial_{\theta}+\mathcal{F}(r, \theta) \partial_{r}$ of Corollary 28 and any (for the moment) real function $v(r, \theta)$ belonging to the set of $T$-periodic functions of $\theta$. Taking the positive anti-clock-wise orientation in $\partial D$, we shall formally use Green's theorem in the domain $D$ to the scaled vector field $\overline{\mathcal{X}} / v$ with associated differential 
$1-$ form $\Omega=(\mathcal{F} d \theta-d r) / v$ :

$$
\iint_{D} \operatorname{div}\left(\frac{\overline{\mathcal{X}}}{v}\right) d r d \theta=\int_{\Gamma_{r_{0}}} \Omega+\int_{\ell_{T}} \Omega+\int_{\Gamma_{r_{0}+\Delta r_{0}}} \Omega+\int_{\ell_{0}} \Omega
$$

Clearly $\int_{\Gamma_{r_{0}}} \Omega=\int_{\Gamma_{r_{0}+\Delta r_{0}}} \Omega=0$ since $\left.\Omega\right|_{\Gamma_{r_{0}}}=\left.\Omega\right|_{\Gamma_{r_{0}+\Delta r_{0}}} \equiv 0$. Moreover

$$
\int_{\ell_{0}} \Omega=-\int_{r_{0}+\Delta r_{0}}^{r_{0}} \frac{d r}{v(r, 0)}, \quad \int_{\ell_{T}} \Omega=-\int_{\Pi\left(r_{0}\right)}^{\Pi\left(r_{0}+\Delta r_{0}\right)} \frac{d r}{v(r, T)} .
$$

Taking also into account that $v(r, 0)=v(r, T)$, equation (40) becomes

$$
\int_{0}^{T} d \theta \int_{\Psi\left(\theta ; r_{0}\right)}^{\Psi\left(\theta ; r_{0}+\Delta r_{0}\right)} \operatorname{div}\left(\frac{\overline{\mathcal{X}}}{v}\right) d r=\mathcal{P}\left(r_{0}+\Delta r_{0}\right)-\mathcal{P}\left(r_{0}\right)+\mathcal{P}\left(\Pi\left(r_{0}\right)\right)-\mathcal{P}\left(\Pi\left(r_{0}+\Delta r_{0}\right)\right),
$$

where $\mathcal{P}(r)$ is a primitive of $\frac{1}{v(r, 0)}$.

Regarding the right-hand side of (41) we note that

$$
\begin{aligned}
\lim _{\Delta r_{0} \rightarrow 0} \frac{\mathcal{P}\left(r_{0}+\Delta r_{0}\right)-\mathcal{P}\left(r_{0}\right)}{\Delta r_{0}} & =\mathcal{P}^{\prime}\left(r_{0}\right)=\frac{1}{v\left(r_{0}, 0\right)}, \\
\lim _{\Delta r_{0} \rightarrow 0} \frac{\mathcal{P}\left(\Pi\left(r_{0}\right)\right)-\mathcal{P}\left(\Pi\left(r_{0}+\Delta r_{0}\right)\right)}{\Delta r_{0}} & =-\frac{d}{d r}(\mathcal{P} \circ \Pi)\left(r_{0}\right)=-\mathcal{P}^{\prime}\left(\Pi\left(r_{0}\right)\right) \Pi^{\prime}\left(r_{0}\right) \\
& =-\frac{\Pi^{\prime}\left(r_{0}\right)}{v\left(\Pi\left(r_{0}\right), 0\right)} .
\end{aligned}
$$

From (24) it follows that

$$
\Theta(r, \theta)=\Theta_{0}(\theta) r+O\left(r^{2}\right)
$$

with $\Theta_{0}(\theta)=1+\omega \operatorname{Cs}^{2} \theta \operatorname{Sn} \theta>0$. We consider the series $W(x, y)=x^{4}+\omega x^{2} y+$ $2 y^{2}+\cdots$ of Theorem 1 and now we define the function $v(r, \theta)$ via the relation $(27)$

$$
v(r, \theta)=\frac{W\left(r \operatorname{Cs} \theta, r^{2} \operatorname{Sn} \theta\right)}{r^{2} \Theta(r, \theta)}=\frac{\Theta_{0}(\theta) r^{4}+O\left(r^{5}\right)}{r^{2}\left(\Theta_{0}(\theta) r+O\left(r^{2}\right)\right)}=r+\sum_{k \geq 2} c_{k}(\theta) r^{k} .
$$

Remark 29. Notice that if $\hat{\eta}_{j}=0$ for any $j \in \mathbb{N}$ then $v(r, \theta)$ is an inverse integrating factor of $\overline{\mathcal{X}}$, that is $\operatorname{div}(\overline{\mathcal{X}} / v) \equiv 0$, hence the fundamental relation $(28)$ can be applied and both limits in (42) give the same result. Thus (41) is a trivial equality.

From the Taylor expansions $\Pi\left(r_{0}\right)=r_{0}+\sum_{k \geq 2} v_{k} r_{0}^{k}$ and (44) we obtain that

$$
\frac{1}{v\left(r_{0}, 0\right)}-\frac{\Pi^{\prime}\left(r_{0}\right)}{v\left(\Pi\left(r_{0}\right), 0\right)}=\sum_{k \geq 0} g_{k} r_{0}^{k}
$$

with $g_{0}=-v_{2}$ and, for any $k \geq 1$,

$$
g_{k}=-(k+1) v_{k+2}
$$

provided that $v_{2}=\cdots=v_{k+1}=0$.

Now we shall analyze the integrand $\operatorname{div}(\overline{\mathcal{X}} / v)$ in $(41)$. We have

$$
\operatorname{div}\left(\frac{\overline{\mathcal{X}}}{v}\right)=\frac{v \operatorname{div}(\overline{\mathcal{X}})-\overline{\mathcal{X}}(v)}{v^{2}}=-\frac{r^{4} \operatorname{Cs}^{4} \theta \sum_{k \geq 1} \hat{\eta}_{k}(\omega, \nu) r^{2 k} \operatorname{Cs}^{2 k} \theta}{r^{2} \Theta^{2}(r, \theta) v^{2}(r, \theta)}
$$


where we have used Corollary 28 in the last equality. Inserting (44) and (43) into (47) leads to

$$
\operatorname{div}\left(\frac{\overline{\mathcal{X}}}{v}\right)=\sum_{k \geq 0} f_{k}(\theta) r^{k},
$$

where $f_{0}(\theta)=-\operatorname{Cs}^{6} \theta \hat{\eta}_{1}(\omega, \nu) / \Theta_{0}^{2}(\theta)$ and

$$
f_{0}(\theta)=\cdots=f_{2 j-1}(\theta) \equiv 0 \text { whereas } f_{2 j}(\theta)=-\operatorname{Cs}^{2(j+3)} \theta \hat{\eta}_{j+1}(\omega, \nu) / \Theta_{0}^{2}(\theta)
$$

provided that $\hat{\eta}_{1}=\cdots=\hat{\eta}_{j}=0$. The left-hand side of (41) becomes

$$
\begin{aligned}
& \int_{0}^{T} d \theta \int_{\Psi\left(\theta ; r_{0}\right)}^{\Psi\left(\theta ; r_{0}+\Delta r_{0}\right)} \operatorname{div}\left(\frac{\overline{\mathcal{X}}}{v}\right) d r=\sum_{k \geq 0} \int_{0}^{T} f_{k}(\theta) d \theta \int_{\Psi\left(\theta ; r_{0}\right)}^{\Psi\left(\theta ; r_{0}+\Delta r_{0}\right)} r^{k} d r \\
= & \sum_{k \geq 0} \frac{1}{k+1} \int_{0}^{T} f_{k}(\theta)\left(\Psi^{k+1}\left(\theta ; r_{0}+\Delta r_{0}\right)-\Psi^{k+1}\left(\theta ; r_{0}\right)\right) d \theta .
\end{aligned}
$$

Therefore

$$
\begin{aligned}
\lim _{\Delta r_{0} \rightarrow 0} \frac{1}{\Delta r_{0}} \int_{0}^{T} d \theta \int_{\Psi\left(\theta ; r_{0}\right)}^{\Psi\left(\theta ; r_{0}+\Delta r_{0}\right)} \operatorname{div}\left(\frac{\overline{\mathcal{X}}}{v}\right) d r & =\sum_{k \geq 0} \frac{1}{k+1} \int_{0}^{T} f_{k}(\theta) \frac{\partial \Psi^{k+1}}{\partial r}\left(\theta ; r_{0}\right) d \theta \\
& =\sum_{k \geq 0} \int_{0}^{T} f_{k}(\theta) \Psi^{k}\left(\theta ; r_{0}\right) \frac{\partial \Psi}{\partial r}\left(\theta ; r_{0}\right) d \theta \\
& =\sum_{k \geq 0} h_{k} r_{0}^{k}
\end{aligned}
$$

where the expansion $\Psi\left(\theta ; r_{0}\right)=r_{0}+\sum_{j \geq 2} \Psi_{j}(\theta) r_{0}^{j}$ was applied in the last equality. The first coefficients $h_{k}$ are

$$
\begin{aligned}
h_{0} & =\int_{0}^{T} f_{0}(\theta) d \theta, \\
h_{1} & =\int_{0}^{T}\left(f_{1}(\theta)+2 f_{0}(\theta) \Psi_{2}(\theta)\right) d \theta, \\
h_{2} & =\int_{0}^{T}\left(f_{2}(\theta)+3 f_{1}(\theta) \Psi_{2}(\theta)+3 f_{0}(\theta) \Psi_{3}(\theta)\right) d \theta .
\end{aligned}
$$

From these expressions and using (49) we get that $h_{0}=\beta_{0} \hat{\eta}_{1}(\omega, \nu)$ and

$$
h_{0}=\cdots=h_{2 j-1}=0 \text { whereas that } h_{2 j}=\beta_{j} \hat{\eta}_{j+1}(\omega, \nu)
$$

provided that $\hat{\eta}_{1}=\cdots=\hat{\eta}_{j}=0$. Here we have defined

$$
\beta_{j}=-\int_{0}^{T} \frac{\operatorname{Cs}^{2(j+3)} \theta}{\Theta_{0}^{2}(\theta)} d \theta<0
$$

for any $j \in \mathbb{N}$.

Dividing both sides in (41) by $\Delta r_{0}$ and taking the limit when $\Delta r_{0} \rightarrow 0$ (using (42) and (45) for the right-hand side of (41) and (50) for the left-hand side of (41)) gives

$$
\sum_{k \geq 0} h_{k} r_{0}^{k}=\sum_{k \geq 0} g_{k} r_{0}^{k}
$$


Now we are going to equate the coefficients in the above series and use (46) and (51) together with Remark 24. The result is that, for any $j \in \mathbb{N}$,

$$
-(2 j+1) v_{2 j}=\beta_{j-1} \hat{\eta}_{j}(\omega, \nu)
$$

provided $\hat{\eta}_{1}=\cdots=\hat{\eta}_{j-1}=0$. The proof is done after defining the positive constants $\alpha_{j}=-\beta_{j-1} /(2 j+1)$.

\subsection{Proof of Theorem 17.}

Proof. Reversing the time $t \mapsto-t$ and denoting $\omega=-k$ family (12) is written in the Andreev canonical form (3) with parameters $\omega$ and $\nu=(a, b, c) \in \mathbb{R}^{3}$. We compute for this polynomial family the first integrability focus quantities $\hat{\eta}_{j} \in \mathbb{R}(\omega)[\nu]$ up to positive multiplicative constants. The outcome is the following one:

$$
\begin{aligned}
\hat{\eta}_{1}(\omega, \nu)= & a \omega, \\
\hat{\eta}_{2}(\omega, \nu)= & \left(2835 a b-8505 c-54 a^{3} \omega-840 a b \omega^{2}+1755 c \omega^{2}-10 a^{3} \omega^{3}+\right. \\
& \left.45 a b \omega^{4}-90 c \omega^{4}\right) /\left(\omega^{2}-9\right), \\
\hat{\eta}_{3}(\omega, \nu)= & \left(2540160 a^{3} b+68040 a^{2} c+44172 a^{5} \omega-9972900 a b^{2} \omega+18295200 b c \omega-\right. \\
& 1185240 a^{3} b \omega^{2}+321165 a^{2} c \omega^{2}-13856 a^{5} \omega^{3}+2732100 a b^{2} \omega^{3}- \\
& 5293575 b c \omega^{3}+75470 a^{3} b \omega^{4}+26405 a^{2} c \omega^{4}-700 a^{5} \omega^{5}-254625 a b^{2} \omega^{5}+ \\
& \left.503475 b c \omega^{5}+910 a^{3} b \omega^{6}-7070 a^{2} c \omega^{6}+7875 a b^{2} \omega^{7}-15750 b c \omega^{7}\right) / \\
& \left(\left(32-3 \omega^{2}\right)\left(\omega^{2}-9\right)\right) .
\end{aligned}
$$

Notice that the functions $\hat{\eta}_{j}$ are well defined for any $\omega \in \Omega$ defined in (4) as it must be. The following definition will be used from now.

Definition 30. We define $\tilde{\eta}_{j}=\hat{\eta}_{j} \bmod \hat{\mathcal{I}}_{j-1}$ in the ring $\mathbb{R}(\omega)[\nu]$. In other words, we let $\tilde{\eta}_{j}$ denote the remainder of $\hat{\eta}_{j}$ upon division by a Gröbner basis of the ideal $\hat{\mathcal{I}}_{j-1}$.

In this example we obtain

$$
\begin{aligned}
& \tilde{\eta}_{1}(\omega, \nu)=a \omega, \\
& \tilde{\eta}_{2}(\omega, \nu)=c\left(21-2 \omega^{2}\right), \\
& \tilde{\eta}_{j}(\omega, \nu)=0 \text { for } j=3,4 .
\end{aligned}
$$

Since the polynomial $21-2 \omega^{2}$ has no roots on $\Omega$, it is clear that $\mathbf{V}_{\mathscr{C}} \cap \mathcal{E}=\mathbf{V}_{\mathbb{R}}\left(\mathcal{G}_{2}\right) \cap \mathcal{E}$.

In order to achieve parts (i) and (ii) we shall use Theorem 15 . The center variety only has one irreducible component $C=\{a=c=0\}$ of $\operatorname{codim}(C)=2$. Moreover the differential of the map

$$
(\omega, \nu) \mapsto G_{2}(\omega, \nu)=\left(\tilde{\eta}_{1}(\omega, \nu), \tilde{\eta}_{2}(\omega, \nu)\right)
$$

at any point $P=(\omega, 0, b, 0) \in C$ is

$$
d_{P} G_{2}=\left(\begin{array}{cccc}
a & \omega & 0 & 0 \\
0 & 0 & 0 & 21-2 \omega^{2}
\end{array}\right) .
$$

It follows that $\operatorname{rank}\left(d_{P} G_{2}\right)=2$ since $\omega \in \Omega$ and in consequence statement (i) holds by Theorem 15 .

Since $\mathbf{V}_{\mathscr{C}} \cap \mathcal{E}=\mathbf{V}_{\mathbb{R}}\left(\mathcal{G}_{2}\right) \cap \mathcal{E}$ we know that 2 is an upper bound of the order of any foci, hence the cyclicity of any focus of (12) at the origin is bounded by 1 . 
Indeed this bound is sharp and we claim that there are perturbations from a focus of order 2 when $(\omega, \nu)=\left(\omega^{\dagger}, \nu^{\dagger}\right)=\left(\omega^{\dagger}, 0, b^{\dagger}, c^{\dagger}\right)$ with $c^{\dagger} \neq 0$ (so that $\tilde{\eta}_{2}\left(\omega^{\dagger}, \nu^{\dagger}\right)=0$ but $\left.\tilde{\eta}_{2}\left(\omega^{\dagger}, \nu^{\dagger}\right) \neq 0\right)$ producing 1 small amplitude limit cycle. Let us perturb along the curve $\varepsilon \mapsto \gamma(\varepsilon)=(\omega(\varepsilon), \nu(\varepsilon))=\left(\omega^{\dagger}, \varepsilon, b^{\dagger}, c^{\dagger}\right) \subset \mathcal{E} \subset \mathbb{R}^{4}$ with $\gamma(0)=\left(\omega^{\dagger}, \nu^{\dagger}\right)$ with $|\varepsilon| \ll 11$. Then $\tilde{\eta}_{1}(\gamma(\varepsilon))=K_{1} \varepsilon+O\left(\varepsilon^{2}\right)$ and $\tilde{\eta}_{2}(\gamma(\varepsilon))=-K_{2}+O(\varepsilon)$ with constants $K_{1}=\omega^{\dagger}$ and $K_{2}=-c^{\dagger}\left(2\left(\omega^{\dagger}\right)^{2}-21\right)$. Choosing $c^{\dagger} \neq 0$ and $\omega^{\dagger} \in \Omega$ such that $K_{1}, K_{2}>0$ we get $\left|\tilde{\eta}_{1}(\gamma(\varepsilon))\right| \ll\left|\tilde{\eta}_{2}(\gamma(\varepsilon))\right| \neq 0$ and $\tilde{\eta}_{1}(\gamma(\varepsilon)) \tilde{\eta}_{2}(\gamma(\varepsilon))<0$. Therefore the claim follows applying elementary bifurcation theory as in Remark 16 and statement (ii) is proved.

\subsection{Proof of Theorem 18.}

Proof. The first integrability focus quantities, up to positive multiplicative constants are

$$
\begin{aligned}
\hat{\eta}_{1}(\omega, \nu)= & -5 E-2 A \omega+3 D \omega+F \omega \\
\hat{\eta}_{2}(\omega, \nu)= & \left(-189 A^{2} E+567 A D E-1134 D^{2} E-756 A E F+1134 D E F+\right. \\
& 1134 E F^{2}+54 A^{3} \omega+243 A^{2} D \omega-1701 A D^{2} \omega-40 A E^{2} \omega+ \\
& 160 D E^{2} \omega-621 A^{2} F \omega+162 A D F \omega-567 D^{2} F \omega-280 E^{2} F \omega+ \\
& 783 A F^{2} \omega-648 D F^{2} \omega-243 F^{3} \omega+45 A^{2} E \omega^{2}+265 A D E \omega^{2}+ \\
& 30 D^{2} E \omega^{2}-160 A E F \omega^{2}+90 D E F \omega^{2}-10 E F^{2} \omega^{2}+10 A^{3} \omega^{3}+ \\
& 45 A^{2} D \omega^{3}+45 A D^{2} \omega^{3}+5 A^{2} F \omega^{3}+50 A D F \omega^{3}+15 D^{2} F \omega^{3}- \\
& \left.35 A F^{2} \omega^{3}+20 D F^{2} \omega^{3}+15 F^{3} \omega^{3}\right) /\left(\omega^{2}-9\right) .
\end{aligned}
$$

Now we reduce $\tilde{\eta}_{j}=\hat{\eta}_{j} \bmod \hat{\mathcal{I}}_{j-1}$ in the ring $\mathbb{R}(\omega)[\nu]$ (see Definition 30) and we obtain

$$
\begin{aligned}
& \tilde{\eta}_{1}(\omega, \nu)=-5 E-2 A \omega+3 D \omega+F \omega, \\
& \tilde{\eta}_{2}(\omega, \nu)=(-E+D \omega)\left(-10 E^{2}+5 D E \omega-5 E F \omega+9 D^{2} \omega^{2}+10 D F \omega^{2}+F^{2} \omega^{2}\right) / \omega^{2}, \\
& \tilde{\eta}_{3}(\omega, \nu)=E(E-D \omega)(2 E+F \omega)\left(-13 E^{2}+17 D E \omega-E F \omega+4 D F \omega^{2}\right) / \omega^{4}, \\
& \tilde{\eta}_{4}(\omega, \nu)=E F(E-D \omega)(E-F \omega)(2 E+F \omega)(3 E+F \omega)(31 E+16 F \omega) / \omega^{5}, \\
& \tilde{\eta}_{j}(\omega, \nu) \equiv 0, \text { for } j=5,6,7 .
\end{aligned}
$$

Using the notation of Definition 7 we check that $g_{4} \notin \sqrt{\mathcal{G}_{3}}$ and $\sqrt{\mathcal{G}_{4}} \neq \mathcal{G}_{4}$. To solve the center problem we use the routine minAssChar in the PRIMDEC.lib library of Singular ([12]) to obtain the prime decomposition

$$
\sqrt{\mathcal{G}_{4}}=\cap_{j=1}^{8} \mathcal{J}_{j}
$$

where

$$
\begin{aligned}
\mathcal{J}_{1} & =\langle 6 D+F, 2 A-3 F, \omega F+2 E\rangle, \\
\mathcal{J}_{2} & =\langle 3 D-2 F, A+F, \omega F-E\rangle, \\
\mathcal{J}_{3} & =\langle 2 A+2 D-F, \omega D-E\rangle, \\
\mathcal{J}_{4} & =\langle 3 D+2 F, 4 A-3 F, \omega F+2 E\rangle, \\
\mathcal{J}_{5} & =\langle 3 D+2 F, 3 A-F, \omega F+3 E\rangle, \\
\mathcal{J}_{6} & =\langle E, 9 D+F, 3 A-F\rangle, \\
\mathcal{J}_{7} & =\langle E, D+F, A+F\rangle, \\
\mathcal{J}_{8} & =\langle E, \omega\rangle .
\end{aligned}
$$


Notice that $C_{j}=\mathbf{V}_{\mathbb{R}}\left(\mathcal{J}_{j}\right)$. Therefore, we get

$$
\mathbf{V}_{\mathbb{R}}\left(\mathcal{G}_{4}\right) \cap \mathcal{E}=\mathbf{V}_{\mathbb{R}}\left(\sqrt{\mathcal{G}_{4}}\right) \cap \mathcal{E}=\left(\cup_{j=1}^{7} \mathbf{V}_{\mathbb{R}}\left(\mathcal{J}_{j}\right)\right) \cap \mathcal{E}=\left(\cup_{j=1}^{7} C_{j}\right) \cap \mathcal{E}
$$

where we have taken into account that $\mathbf{V}_{\mathbb{R}}\left(\mathcal{J}_{8}\right) \cap \mathcal{E}=\emptyset$. Notice that any member of family (14) belongs to the trivial center strata defined by the Hamiltonian fields or the time-reversible with respect some coordinate axis, hence to know whether $(\omega, \nu) \in C_{j} \cap \mathcal{E}$ for $j \in\{1, \ldots, 7\}$ implies $(\omega, \nu) \in \mathbf{V}_{\mathscr{C}} \cap \mathcal{E}$ is, in general, a difficult problem. To solve it we will use the ideas explained in the following remark.

Remark 31. System (14) is a particular case of system

$$
\dot{x}=y P_{3}(x), \quad \dot{y}=P_{0}(x)+P_{1}(x) y+P_{2}(x) y^{2}
$$

with $P_{3}(0) \neq 0$. Performing the (analytic and tangent to the identity) Cherkas change of variables $(x, y) \mapsto(x, y / \xi(x))$ with

$$
\xi(x)=\exp \left(\int_{0}^{x} \frac{P_{2}(\sigma)}{P_{3}(\sigma)} d \sigma\right),
$$

and rescaling the time (dividing the vector field by $P_{3}(x) \xi(x)$ ), system (53) is transformed into the Liénard system

$$
\dot{x}=y, \quad \dot{y}=g(x)+y f(x)
$$

with

$$
f(x)=\frac{P_{1}(x)}{P_{3}(x) \xi(x)}, \quad g(x)=\frac{P_{0}(x)}{P_{3}(x) \xi^{2}(x)} .
$$

When (53) comes from (14) we have $P_{3}(x)=-1+A x, P_{0}(x)=x^{3}+D x^{4}, P_{1}(x)=$ $\omega x+E x^{2}$ and $P_{2}(x)=F$. This choice gives $\xi(x)=(1-A x)^{F / A}$ if $A \neq 0$ and $\xi(x)=\exp (-F x)$ when $A=0$ (which is just the limit when $A \rightarrow 0$ of $\xi(x)$ ) and consequently for any value of $A$ we get

$$
g(x)=-x^{3}+\cdots, f(x)=-\omega x+\cdots .
$$

In Theorem 2.6 of [22] the authors make a generalization of a result of Cherkas [9] that characterizes the nondegenerate centers of Liénard equations to some class of degenerate singularities including our nilpotent class where $f$ and $g$ are analytic functions with starting terms like in (55). In our context their result is stated as follows: The origin of the analytic Liénard system with $f$ and $g$ as in (55) has a (nilpotent) center at the origin if and only if, for any $x$ small enough, the system

$$
\mathcal{F}(x)=\mathcal{F}(z), \quad \mathcal{G}(x)=\mathcal{G}(z),
$$

has a unique solution $z(x)$ satisfying $z(0)=0$ and $z^{\prime}(0)<0$ where we have defined

$$
\mathcal{F}(x)=\int_{0}^{x} f(\sigma) d \sigma, \quad \mathcal{G}(x)=\int_{0}^{x} g(\sigma) d \sigma .
$$

Now we continue the proof using the technique in Remark 31 to family (14) when the parameters lie on $\mathbf{V}_{\mathbb{R}}\left(\mathcal{J}_{j}\right) \cap \mathcal{E}$ for $j=1, \ldots, 7$.

Let $(\omega, \nu) \in C_{1}$, that is, $D=-F / 6, A=3 F / 2$ and $E=-\omega F / 2$. Then

$$
\begin{aligned}
\mathcal{F}(x) & =\frac{\omega}{4 F^{2}}\left(4-\frac{2^{2 / 3}(2-F x)^{2}}{(2-3 F x)^{2 / 3}}\right), \\
\mathcal{G}(x) & =\frac{48-96 F x+24 F^{2} x^{2}+8 F^{3} x^{3}-F^{4} x^{4}}{6 \times 2^{2 / 3} F^{4}(2-3 F x)^{4 / 3}}-\frac{2}{F^{4}},
\end{aligned}
$$


and the solution of $(56)$ is

$z(x)=\frac{-12+F x(20-3 F x)+\sqrt{3(-6+F x)}(-2+F x) \sqrt{-2+3 F x}}{2 F(-2+3 F x)}=-x+O\left(x^{2}\right)$,

hence $C_{1} \subset \mathbf{V}_{\mathscr{C}}$.

If $(\omega, \nu) \in C_{2}$ we take $D=2 F / 3, A=-F$ and $E=\omega F$. Then

$$
\mathcal{F}(x)=-\frac{\omega x^{2}}{6}(3+2 F x), \quad \mathcal{G}(x)=-\frac{x^{4}}{36}(3+2 F x)^{2},
$$

and the solution of (56) is

$$
z(x)=\frac{-3-2 F x+\sqrt{3\left(3-4 F x-4 F^{2} x^{2}\right)}}{4 F}=-x+O\left(x^{2}\right),
$$

proving that $C_{2} \subset \mathbf{V}_{\mathscr{C}}$.

Let $(\omega, \nu) \in C_{3}$ so that $F=2(A+D)$ and $E=\omega D$. Then

$$
\mathcal{F}(x)=-\frac{1}{2} \omega x^{2}(1-A x)^{-2(A+D) / A}, \quad \mathcal{G}(x)=-\frac{\mathcal{F}^{2}(x)}{\omega^{2}} .
$$

We note that system $(56)$ is written locally around $(x, z)=(0,0)$ as the analytic equations

$$
\Delta(z, x)=x^{2}-z^{2}+\cdots=0, \quad \Delta^{2}(z, x)=0,
$$

where the dots are higher order terms. Therefore we only need to analyze the local structure of the solutions of the first equation. There are several methods to do that and we opt by the way of singularities of smooth maps, see for example the book [23]. Using subscripts for partial derivatives, $\Delta(0,0)=\Delta_{z}(0,0)=0$ hence the point $(z, x)=(0,0)$ is called a singularity of the map $\Delta$ because the hypothesis of the Implicit Function Theorem fail at the singularity. We say that the maps $\Delta(z, x)$ and $\hat{\Delta}(z, x)$ are equivalent if there exist a local diffeomorphism of $\mathbb{R}^{2}$ of the form $(z, x) \mapsto(Z(z, x), X(x))$ mapping the origin to $(0,0)$ and a positive function $U(z, x)$ such that $U(z, x) \Delta(Z(z, x), X(x))=\hat{\Delta}(z, x)$ and where the diffeomorphism preserves the orientations of $z$ and $x$, that is, the derivatives $Z_{z}(z, x)>0$ and $X_{x}(x)>0$. We call $\hat{\Delta}$ a normal form of $\Delta$ when it is the simplest representative from a whole equivalence class of mappings. Let $D^{2} \Delta(0,0)$ stands for the Hessian matrix of $\Delta$ at $(0,0)$. Since in our case $\Delta_{z z}(0,0)>0, \Delta_{x}(0,0)=0$, and $\operatorname{det}\left(D^{2} \Delta(0,0)\right)<0$ then the singularity is called simple and the normal form is just $\hat{\Delta}(z, x)=x^{2}-z^{2}$. This proves that the equation $\Delta(z, x)=0$ only has two real solutions in a neighborhood of the origin having the form $x+O\left(x^{2}\right)$ and $-x+O\left(x^{2}\right)$, hence $C_{3} \subset \mathbf{V}_{\mathscr{C}}$.

Let $(\omega, \nu) \in C_{4}$ so that $D=-2 F / 3, A=3 F / 4$ and $E=-\omega F / 2$. Then

$$
\begin{aligned}
\mathcal{F}(x) & =\frac{4 \omega}{F^{2}}-\frac{4 \times 2^{2 / 3} \omega(-2+F x)^{2}}{F^{2}(4-3 F x)^{4 / 3}}, \\
\mathcal{G}(x) & =-\frac{32}{F^{4}}-\frac{64 \times 2^{1 / 3}(-2+F x)(24+F x(-36+F x(12+F x)))}{3 F^{4}(4-3 F x)^{8 / 3}},
\end{aligned}
$$

and the solution of (56) is

$$
z(x)=-\frac{2\left(-6+F x(13-6 F x)+\sqrt{3} \sqrt{(-2+F x)^{2}(3-2 F x)}\right)}{F(4-3 F x)^{2}}=-x+O\left(x^{2}\right),
$$

proving that $C_{4} \subset \mathbf{V}_{\mathscr{C}}$. 
Let $(\omega, \nu) \in C_{5}$, hence $D=-2 F / 3, A=F / 3$ and $E=-\omega F / 3$. Then

$\mathcal{F}(x)=-\frac{9 \omega}{2 F^{2}}-\frac{27 \omega(-3+2 F x)}{2 F^{2}(-3+F x)^{2}}, \mathcal{G}(x)=-\frac{27}{4 F^{4}}+\frac{729(3-2 F x)^{2}(3+F x(-2+F x))}{4 F^{4}(-3+F x)^{6}}$,

and the solution of (56) is

$$
z(x)=\frac{3 x}{-3+2 F x}=-x+O\left(x^{2}\right),
$$

proving that $C_{5} \subset \mathbf{V}_{\mathscr{C}}$.

Let $(\omega, \nu) \in C_{6}$, hence $E=0, D=-F / 9$ and $A=F / 3$. Then

$$
\begin{aligned}
\mathcal{F}(x) & =-\frac{3 \omega}{2 F^{2}}+\frac{81 \omega(-1+F x)}{2 F^{2}(-3+F x)^{3}}, \\
\mathcal{G}(x) & =-\frac{9}{4 F^{4}}+\frac{243(-1+F x)(-27+F x(27+2 F x(-9+F x)))}{4 F^{4}(-3+F x)^{6}},
\end{aligned}
$$

and the solution of (56) is

$$
z(x)=\frac{-9+10 F x-F^{2} x^{2}+(3-F x) \sqrt{(-9+F x)(-1+F x)}}{2 F(-1+F x)}=-x+O\left(x^{2}\right),
$$

proving that $C_{6} \subset \mathbf{V}_{\mathscr{C}}$.

Let $(\omega, \nu) \in C_{7}$, hence $E=0, D=-F$ and $A=-F$. Then

$$
\mathcal{F}(x)=-\frac{\omega x^{2}}{2}, \quad \mathcal{G}(x)=\frac{x^{4}}{12}\left(-3+2 F^{2} x^{2}\right),
$$

are even functions and therefore the solution of (56) is the trivial one $z=-x$ and so $C_{7} \subset \mathbf{V}_{\mathscr{C}}$. This computation solves the center problem at the origin of family (14) which is the first part of the theorem.

As a byproduct of $\mathbf{V}_{\mathscr{C}} \cap \mathcal{E}=\mathbf{V}_{\mathbb{R}}\left(\mathcal{G}_{4}\right) \cap \mathcal{E}$, the maximum order of a focus at the origin is 4 . Actually it is easy to check (using resultants for example) that there is no foci of order 4 . We are going to prove that indeed we can made bifurcations with 2 limit cycles from the focus of order 3 when $(\omega, \nu)=\left(\omega^{\dagger}, \nu^{\dagger}\right)$ with

$$
\left(\omega^{\dagger}, \nu^{\dagger}\right)=\left(\omega^{\dagger}, A^{\dagger}, D^{\dagger}, E^{\dagger}, F^{\dagger}\right)=\left(1,1, \frac{1}{42}(-35+\sqrt{385}), \frac{1}{70}(-35+\sqrt{385}), 2\right) .
$$

We get $\tilde{\eta}_{j}\left(\omega^{\dagger}, \nu^{\dagger}\right)=0$ for $j=1,2,3$, but $\tilde{\eta}_{4}\left(\omega^{\dagger}, \nu^{\dagger}\right) \neq 0$ and perturbing along the curve $\varepsilon \mapsto \gamma(\varepsilon)=(\omega(\varepsilon), \nu(\varepsilon)) \subset \mathcal{E} \subset \mathbb{R}^{5}$ with $\gamma(0)=\left(\omega^{\dagger}, \nu^{\dagger}\right)$ defined by

$$
\varepsilon \mapsto\left(\omega^{\dagger}, A^{\dagger}, D^{\dagger}, E^{\dagger}+\frac{63}{2(-217+11 \sqrt{385})} \varepsilon-\frac{1}{5} \varepsilon^{2}, F^{\dagger}+\frac{315}{2(-217+11 \sqrt{385})} \varepsilon\right)
$$

with $\varepsilon$ sufficiently small. On this curve we have $\tilde{\eta}_{1}(\gamma(\varepsilon))=K_{1} \varepsilon^{2}+O\left(\varepsilon^{3}\right), \tilde{\eta}_{2}(\gamma(\varepsilon))=$ $-K_{2} \varepsilon+O\left(\varepsilon^{2}\right)$, and $\tilde{\eta}_{3}(\gamma(\varepsilon))=K_{3}+O(\varepsilon)$, for some constants $K_{j}>0$ and therefore by the arguments of Remark 16 we get that 2 small amplitude limit cycles can be made to bifurcate from the focus at the origin when $(\omega, \nu)=\left(\omega^{\dagger}, \nu^{\dagger}\right)$ in family (14). This proves statement (iv).

Now we start the analysis of the cyclicity of the origin inside family (14). We will use Theorem 15. First we claim that

$$
\operatorname{codim}\left(C_{j}\right)=3 \text { when } j \neq 3 \text { and } \operatorname{codim}\left(C_{3}\right)=2 .
$$


The proof of the claim uses the fact that if the map $F: \mathbb{R}^{n} \rightarrow \mathbb{R}^{m}$ with $n \geq m$ is such that its differential $d_{P} F$ at $P \in \mathbb{R}^{n}$ has maximal rank (in other words, $F$ is a submersion at its regular point $P$ ) then the origin of $\mathbb{R}^{m}$ is a regular value of $F$ restricted to some neighborhood $U \subset \mathbb{R}^{n}$ of $P$ and therefore $U \cap F^{-1}(0)$ is a smooth submanifold of $\mathbb{R}^{n}$ of codimension $m$.

Each irreducible component $C_{j}$ of the center variety is defined by $C_{j}=F_{j}^{-1}(0)$ where $F_{j}: \mathbb{R}^{5} \rightarrow \mathbb{R}^{3}$ when $j \neq 3$ and $F_{3}: \mathbb{R}^{5} \rightarrow \mathbb{R}^{2}$ being

$$
\begin{aligned}
& F_{1}(\omega, \nu)=(6 D+F, 2 A-3 F, \omega F+2 E), \\
& F_{2}(\omega, \nu)=(3 D-2 F, A+F, \omega F-E), \\
& F_{3}(\omega, \nu)=(2 A+2 D-F, \omega D-E), \\
& F_{4}(\omega, \nu)=(3 D+2 F, 4 A-3 F, \omega F+2 E), \\
& F_{5}(\omega, \nu)=(3 D+2 F, 3 A-F, \omega F+3 E), \\
& F_{6}(\omega, \nu)=(E, 9 D+F, 3 A-F), \\
& F_{7}(\omega, \nu)=(E, D+F, A+F) .
\end{aligned}
$$

The claim is proved because the differential of $F_{j}$ has maximal rank at any point $(\omega, \nu) \in \mathbb{R}^{5}$ for all subindex $j$.

Now we define the map $(\omega, \nu) \mapsto G_{k}(\omega, \nu)=\left(\tilde{\eta}_{1}(\omega, \nu), \ldots, \tilde{\eta}_{k}(\omega, \nu)\right)$ and we compute, for $k \in\{3,2\}$, the rank of its Jacobian at arbitrary points $P_{j}=(\omega, \nu)$ on each component $C_{j}$. Taking into account that $\omega \in \Omega$ we obtaining the following results:

- If $P_{j} \in C_{j}$ with $j \neq 3$ then $\operatorname{rank}\left(d_{P_{j}} G_{3}\right)=3$ if $P_{j} \neq(\omega, 0,0,0,0)$ and $\operatorname{rank}\left(d_{P_{j}} G_{3}\right)=1$ otherwise.

- $\operatorname{rank}\left(d_{P_{3}} G_{2}\right)=2$ if $P_{3} \in C_{3}$ such that $(A+3 D)(2 A+3 D) \neq 0$ and $\operatorname{rank}\left(d_{P_{3}} G_{2}\right)=1$ otherwise.

The above implies statements (i) and (ii) from Theorem 15. In summary, only the limit cycle bifurcations from the center at the origin when either $\nu=0$ or $A=-3 D, F=-4 D$ and $E=\omega D$ or $A=-3 D / 2, F=-D$ and $E=\omega D$ remains open. Statement (iii) analyze the firs case.

We start the proof of (iii). Arbitrary perturbations within family (14) of the integrable center at the origin when $(\omega, \nu)=(\omega, 0,0,0,0)$ can be analyzed as follows. The unperturbed system $\dot{x}=-y, \dot{y}=x^{3}+\omega x y$ has a global center possessing the inverse integrating factor $V_{0}(x, y ; \omega)=x^{4}+2 y^{2}+\omega x^{2} y$ from which we obtain its first integral

$$
H(x, y ; \omega)=\frac{V_{0}^{1 / 4}(x, y ; \omega)}{\exp \left(\frac{\omega}{2 \sqrt{8-\omega^{2}}} \arctan \left(\frac{\omega x^{2}+4 y}{\sqrt{8-\omega^{2}} x^{2}}\right)\right)} .
$$

We consider an arbitrary deformation $\dot{x}=-y+\varepsilon P(x, y ; \nu, \varepsilon), \dot{y}=x^{3}+\omega x y+$ $\varepsilon Q(x, y ; \nu, \varepsilon)$, with $P(x, y ; \nu, \varepsilon)=a(\varepsilon) x y$ and $Q(x, y ; \nu)=d(\varepsilon) x^{4}+e(\varepsilon) x^{2} y+f(\varepsilon) y^{2}$ with $\nu=(a(0), d(0), e(0), f(0))=(A, D, E, F)$. Taking a transversal to the perturbed trajectories and parameterizing it with the values of $H$, it is known since Poincaré that the expansion of the displacement map near $\varepsilon=0$ is $\delta(h ; \omega, \nu, \varepsilon)=$ 
$M(h ; \omega, \nu) \varepsilon+O\left(\varepsilon^{2}\right)$ and the first Melnikov function has the expression of a generalized Abelian integral

$$
M(h ; \omega, \nu)=\int_{\gamma_{h}} \frac{P(x, y ; \nu, 0) d y-Q(x, y ; \nu, 0) d x}{V_{0}(x, y ; \omega)}
$$

where $\gamma_{h}$ are the family of ovals surrounding the origin contained in the level curves $\left\{H(x, y ; \omega)=h: h \in \mathbb{R}^{+}\right\}$. The reader can consult the book [11] for a modern exposition. Assuming $M \not \equiv 0$ (otherwise higher order bifurcations may occur) and recalling we are only interested in the limit cycle bifurcations from the origin (not in the entire period annulus), we need to study the number of isolated zeroes of the function $M(h ; \omega, \nu)$ for $h>0$ sufficiently small which corresponds with small amplitude limit cycles. One can use the variables $(x, y) \mapsto(\rho, \varphi)$ with $x=\rho \cos \varphi$, $y=\rho^{2} \sin \varphi$ to parameterize by the angle $\varphi$ the line integral defining $M(h ; \omega, \nu)$ and we obtain $M(h ; \omega, \nu)=-42^{3 / 4} \alpha(\omega, \nu) h$ where

$$
\alpha(\omega, \nu)=\int_{0}^{2 \pi} \exp \left[\frac{\omega}{2 \sqrt{8-\omega^{2}}} \arctan \left(\frac{\omega+4 \sec (\varphi) \tan (\varphi)}{\sqrt{8-\omega^{2}}}\right)\right] R(\varphi ; \omega, \nu) d \varphi
$$

with

and

$$
R(\varphi ; \omega, \nu)=\frac{\sin (\varphi)(-3+\cos (2 \varphi) N(\varphi ; \omega, \nu)}{\left[11-4 \cos (2 \varphi)+\cos (4 \varphi)+8 \omega \cos ^{2}(\varphi) \sin (\varphi)\right]^{9 / 4}}
$$

$$
\begin{aligned}
N(\varphi ; \omega, \nu)= & 3(A+D)+4 F+4(A+D-F) \cos (2 \varphi)+(A+D) \cos (4 \varphi)+ \\
& 8(E+A \omega) \cos ^{2}(\varphi) \sin (\varphi) .
\end{aligned}
$$

The linear expression of $M(. ; \omega, \nu)$ finishes the proof.

Remark 32. We are going to apply Theorem 14 to analyze family (14). We know that $\mathbf{V}_{\mathbb{R}}(\mathcal{G}) \cap \mathcal{E}=\mathbf{V}_{\mathbb{R}}\left(\mathcal{G}_{4}\right) \cap \mathcal{E}$. Since we do not know if $\mathbf{V}_{\mathbb{C}}(\mathcal{G})=\mathbf{V}_{\mathbb{C}}\left(\mathcal{G}_{4}\right)$ is true or not we will use Proposition 11 and Remark 12 to check that the equality $\mathbf{V}_{\mathbb{C}}\left(\mathcal{G}^{*}\right)=\mathbf{V}_{\mathbb{C}}\left(\mathcal{G}_{4}^{*}\right)$ holds where $\mathcal{G}_{4}^{*}=\left\langle g_{1}\left(\omega^{*}, \nu\right), \ldots, g_{4}\left(\omega^{*}, \nu\right)\right\rangle$ in the ring $\mathbb{R}[\nu]$ and $\omega$ is fixed to any value $\omega^{*} \in \Omega$.

Let $\hat{F}: \mathbb{C}^{5} \rightarrow \mathbb{C}^{4}$ be the map $(\omega, \nu) \mapsto \hat{F}(\omega, \nu)=\left(g_{1}(\omega, \nu), \ldots, g_{4}(\omega, \nu)\right)$ and $P=$ $(\omega, \nu) \in \mathbb{C}^{5}$. Then, we have the $4 \times 5$ Jacobian matrix $d_{P} \hat{F}$ has $\operatorname{rank}\left(d_{P} \hat{F}\right)=4$ if and only if $P \in \mathbb{C}^{5} \backslash \sigma$ where $\sigma=\mathbf{V}_{\mathbb{C}}\left(\delta_{1}, \ldots, \delta_{5}\right)$ being $\delta_{j}(\omega, \nu) \in \mathbb{R}[\omega, \nu]$ the determinant of the matrix $d_{P} \hat{F}$ whose $j$ th column has been removed. So $\operatorname{rank}\left(d_{P} \hat{F}\right)=4$ at $P \in \mathbf{V}_{\mathbb{C}}\left(\mathcal{G}_{4}\right) \backslash \Sigma$ where $\Sigma=\mathbf{V}_{\mathbb{C}}\left(\mathcal{G}_{4}\right) \cap \sigma=\mathbf{V}_{\mathbb{C}}(\mathcal{K})$ and $\mathcal{K}=\left\langle g_{1}, \ldots, g_{4}, \delta_{1}, \ldots, \delta_{5}\right\rangle$. Straightforward computations with Singular yield that $\delta_{j} \in \sqrt{\mathcal{G}_{4}}$ for all $j$, hence $\sqrt{\mathcal{K}}=\sqrt{\mathcal{G}_{4}}$. In conclusion, $\Sigma=\mathbf{V}_{\mathbb{C}}(\mathcal{K})=\mathbf{V}_{\mathbb{C}}\left(\mathcal{G}_{4}\right)$ and therefore $\mathbf{V}_{\mathbb{C}}\left(\mathcal{G}_{4}\right) \backslash \Sigma=\emptyset$ so that $\overline{\mathbf{V}_{\mathbb{C}}\left(\mathcal{G}_{4}\right) \backslash \Sigma} \neq \mathbf{V}_{\mathbb{C}}\left(\mathcal{G}_{4}\right)$ and we cannot apply Proposition 11 to conclude that $\mathbf{V}_{\mathbb{C}}\left(\mathcal{G}_{4}^{*}\right)=\mathbf{V}_{\mathbb{C}}\left(\mathcal{G}^{*}\right)$ neither Theorem 14 .

\subsection{Proof of Theorem 19.}

Proof. The first integrability focus quantities, up to positive multiplicative constants are

$$
\begin{aligned}
\hat{\eta}_{1}(\omega, \nu)= & B \omega-5 A \\
\hat{\eta}_{2}(\omega, \nu)= & \left(1134 A B^{2}-945 A C+8505 D-280 A^{2} B \omega-243 B^{3} \omega+\right. \\
& 675 B C \omega-10 A B^{2} \omega^{2}+5 A C \omega^{2}-1755 D \omega^{2}+15 B^{3} \omega^{3}-55 B C \omega^{3}+ \\
& \left.90 D \omega^{4}\right) /\left(\omega^{2}-9\right) .
\end{aligned}
$$


Now we reduce $\tilde{\eta}_{j}=\hat{\eta}_{j} \bmod \hat{\mathcal{I}}_{j-1}$ in the ring $\mathbb{R}(\omega)[\nu]$ according to Definition 30 and we obtain

$$
\begin{aligned}
\tilde{\eta}_{1}(\omega, \nu)= & B \omega-5 A \\
\tilde{\eta}_{2}(\omega, \nu)= & -525 D+B^{3} \omega-30 B C \omega+50 D \omega^{2}, \\
\tilde{\eta}_{3}(\omega, \nu)= & -2373 B^{2} D-73065 C D-3768 B C^{2} \omega+76 B^{2} D \omega^{2}+6530 C D \omega^{2}, \\
\tilde{\eta}_{4}(\omega, \nu)= & D\left(12163329495 B D+10287910665 C^{2} \omega-4918800384 B D \omega^{2}-\right. \\
& 1187245380 C^{2} \omega^{3}+682946208 B D \omega^{4}+28598500 C^{2} \omega^{5}- \\
& \left.30262360 B D \omega^{6}\right) /\left(\omega\left(-14613+1306 \omega^{2}\right)\right), \\
\tilde{\eta}_{j}(\omega, \nu) \equiv & 0, \text { for } j=5,6,7 .
\end{aligned}
$$

Computing the first polynomial ideals $\mathcal{G}_{k}$ of Definition 7 we check that $g_{4} \notin \sqrt{\mathcal{G}_{3}}$ and $\sqrt{\mathcal{G}_{4}} \neq \mathcal{G}_{4}$. Using again Singular $([12])$ we obtain the prime decomposition

$$
\sqrt{\mathcal{G}_{4}}=\mathcal{J} \cap\left(\cap_{j=1}^{6} \mathcal{J}_{j}\right)
$$

where $\mathcal{J}=\langle A, B, D\rangle$ and each $\mathcal{J}_{j}$ has a generator given by a polynomial $p_{j}(\omega)$ with the property $p_{j}^{-1}(0) \notin \Omega$. Therefore, $\mathbf{V}_{\mathscr{C}}=\mathbf{V}_{\mathbb{R}}(\mathcal{J})$ and

$$
\mathbf{V}_{\mathbb{R}}\left(\mathcal{G}_{4}\right)=\mathbf{V}_{\mathbb{R}}\left(\sqrt{\mathcal{G}_{4}}\right)=\mathbf{V}_{\mathbb{R}}(\mathcal{J}) \cup \Delta=\mathbf{V}_{\mathscr{C}} \cup \Delta
$$

where $\Delta=\cup_{j=1}^{6} \mathbf{V}_{\mathbb{R}}\left(\mathcal{J}_{j}\right)$, hence satisfying $\Delta \cap \mathcal{E}=\emptyset$. Thus $\mathbf{V}_{\mathscr{C}} \cap \mathcal{E}=\mathbf{V}_{\mathbb{R}}\left(\mathcal{G}_{4}\right) \cap \mathcal{E}$ proving the first part of the theorem. Moreover, from this equality we obtain that the maximum order of a focus at the origin is 4 , hence the cyclicity of the foci is at most 3 and there are examples in [5] with 3 limit cycles proving statement (iii).

Now we are going to prove statements (i) and (ii) by using Theorem 15 . Notice that the center variety only has one irreducible component $\hat{C}=\{A=B=D=0\}$ with $\operatorname{codim}(\hat{C})=3$. We define the map $(\omega, \nu) \mapsto G_{j}(\omega, \nu)=\left(\tilde{\eta}_{1}(\omega, \nu), \ldots, \tilde{\eta}_{j}(\omega, \nu)\right)$ whose Jacobian when $j=3$ at an arbitrary point $P=(\omega, 0,0, C, 0) \in \hat{C}$ is

$$
d_{P} G_{3}=\left(\begin{array}{ccccc}
0 & -5 & \omega & 0 & 0 \\
0 & 0 & \frac{-18}{175} C \omega & 0 & \frac{3}{875}\left(-525+50 \omega^{2}\right) \\
0 & 0 & -\frac{1256}{1575} C^{2} \omega & 0 & \frac{1}{4725} C\left(-73065+6530 \omega^{2}\right)
\end{array}\right) .
$$

Taking into account that $\omega \in \Omega$ we get $\operatorname{rank}\left(d_{P} G_{3}\right)=3$ if $C \neq 0$ and $\operatorname{rank}\left(d_{P} G_{2}\right)=$ 2 if $C=0$. Consequently statements (i) and (ii) follow from Theorem 15 .

Remark 33. Using our notation, the example of [5] with 3 limit cycles needs the four generators of $\hat{\mathcal{I}}_{4}=\left\langle\tilde{\eta}_{1}, \ldots, \tilde{\eta}_{4}\right\rangle$ with $\tilde{\eta}_{j}=\hat{\eta}_{j} \bmod \hat{\mathcal{I}}_{j-1}$ in the ring $\mathbb{R}(\omega)[\nu]$ in such a way that the origin becomes a focus of order 4 at parameter values $(\omega, \nu)=\left(\omega^{\dagger}, \nu^{\dagger}\right) \in E \backslash \mathbf{V}_{\mathscr{C}}$, that is, $\tilde{\eta}_{j}\left(\omega^{\dagger}, \nu^{\dagger}\right)=0$ for $j=1,2,3$, but $\tilde{\eta}_{4}\left(\omega^{\dagger}, \nu^{\dagger}\right) \neq 0$. Then by using the technique explained in Remark 16 we get that 3 small amplitude limit cycles can be made to bifurcate from the focus at the origin for $(\omega, \nu)=\left(\omega^{\dagger}, \nu^{\dagger}\right)$ in family (15). Specifically, the example of [5] comes from the focus with $\left(\omega^{\dagger}, \nu^{\dagger}\right)=\left(\omega^{\dagger}, A^{\dagger}, B^{\dagger}, C^{\dagger}, D^{\dagger}\right)=(1,1 / 5,1,(-95+\sqrt{3041065}) / 16500,(645-$

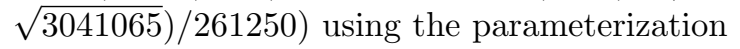

$$
\varepsilon \mapsto(\omega(\varepsilon), \nu(\varepsilon))=\left(\omega^{\dagger}, A^{\dagger}+\varepsilon^{3}, B^{\dagger}, C^{\dagger}-\varepsilon, D^{\dagger}+\frac{6}{95} \varepsilon-\varepsilon^{2}\right)
$$

so that $\tilde{\eta}_{1}(\gamma(\varepsilon))=-K_{1} \varepsilon^{3}, \tilde{\eta}_{2}(\gamma(\varepsilon))=K_{2} \varepsilon^{2}, \tilde{\eta}_{3}(\gamma(\varepsilon))=-K_{3} \varepsilon+O\left(\varepsilon^{2}\right)$, and $\tilde{\eta}_{4}(\gamma(\varepsilon))=K_{4}+O(\varepsilon)$, for some constants $K_{j}>0$. 
Remark 34. In the sequel we prepare the use of Theorem 14 to analyze family (15). We recall that $\mathbf{V}_{\mathbb{R}}(\mathcal{G}) \cap \mathcal{E}=\mathbf{V}_{\mathbb{R}}\left(\mathcal{G}_{4}\right) \cap \mathcal{E}$ from the proof of Theorem 19. Now we move to the complex setting and we want to see if the equality $\mathbf{V}_{\mathbb{C}}(\mathcal{G})=\mathbf{V}_{\mathbb{C}}\left(\mathcal{G}_{4}\right)$ holds. But we lower our pretensions and focus, in the light of Proposition 11 and Remark 12 , into the verification of the weaker equality $\mathbf{V}_{\mathbb{C}}\left(\mathcal{G}^{*}\right)=\mathbf{V}_{\mathbb{C}}\left(\mathcal{G}_{4}^{*}\right)$ where $\mathcal{G}_{4}^{*}=\left\langle g_{1}\left(\omega^{*}, \nu\right), \ldots, g_{4}\left(\omega^{*}, \nu\right)\right\rangle$ in the ring $\mathbb{R}[\nu]$ where $\omega$ takes any fixed value $\omega^{*} \in \Omega$. To apply Proposition 11 we consider the map $F: \mathbb{C}^{5} \rightarrow \mathbb{C}^{4}$ defined by $(\omega, \nu) \mapsto$ $F(\omega, \nu)=\left(g_{1}(\omega, \nu), \ldots, g_{4}(\omega, \nu)\right)$ such that, at any point $P=(\omega, \nu) \in \mathbb{C}^{5}$, we have the $4 \times 5$ Jacobian matrix $d_{P} F$ such that $\operatorname{rank}\left(d_{P} F\right)=4$ at any point $P \in \mathbb{C}^{5} \backslash \sigma$ where $\sigma=\mathbf{V}_{\mathbb{C}}\left(\delta_{1}, \ldots, \delta_{5}\right)$ where $\delta_{j}(\omega, \nu) \in \mathbb{R}[\omega, \nu]$ denotes the determinant of the matrix $d_{P} F$ whose $j$ th column has been removed. In particular, $\operatorname{rank}\left(d_{P} F\right)=4$ at $P \in \mathbf{V}_{\mathbb{C}}\left(\mathcal{G}_{4}\right) \backslash \Sigma$ where $\Sigma=\mathbf{V}_{\mathbb{C}}\left(\mathcal{G}_{4}\right) \cap \sigma=\mathbf{V}_{\mathbb{C}}(\mathcal{K})$ where $\mathcal{K}=\left\langle g_{1}, \ldots, g_{4}, \delta_{1}, \ldots, \delta_{5}\right\rangle$. Straightforward computations with SingULAR yield the prime decomposition $\sqrt{\mathcal{K}}=$ $\mathcal{J} \cap \mathcal{K}_{1}$ where $\mathcal{J}=\langle A, B, D\rangle$ and $\mathcal{K}_{1}=\langle\omega, A, D\rangle$. In conclusion

$$
\Sigma=\mathbf{V}_{\mathbb{C}}(\mathcal{K})=\mathbf{V}_{\mathbb{C}}(\sqrt{\mathcal{K}})=\mathbf{V}_{\mathbb{C}}(\mathcal{J}) \cup \mathbf{V}_{\mathbb{C}}\left(\mathcal{K}_{1}\right)=\mathbf{V}_{\mathbb{C}}\left(\mathcal{J} \cap \mathcal{K}_{1}\right)=\mathbf{V}_{\mathbb{C}}(A, D, \omega B) .
$$

We get that $\Sigma$ is a finite union of intersections of hyperplanes in $\mathbb{C}^{5}$ which intersect transversally with $\mathbf{V}_{\mathbb{C}}\left(\mathcal{G}_{4}\right)$, hence the Zariski closure $\overline{\mathbf{V}_{\mathbb{C}}\left(\mathcal{G}_{4}\right) \backslash \Sigma}=\mathbf{V}_{\mathbb{C}}\left(\mathcal{G}_{4}\right)$. Therefore, for any fixed value $\omega=\omega^{*} \in \Omega$ we have by Proposition 11 that $\mathbf{V}_{\mathbb{C}}\left(\mathcal{G}_{4}^{*}\right)=\mathbf{V}_{\mathbb{C}}\left(\mathcal{G}^{*}\right)$ as claimed.

We cannot apply Theorem 14(i) because $\sqrt{\mathcal{G}_{4}} \neq \mathcal{G}_{4}$. Thus we try to apply Theorem 14(ii) with a fixed (but arbitrary) value $\omega=\omega^{*} \in \Omega$. Using the ring definition ring $r=(0, w)$, ( $A, B, C, D)$ in Singular we find the primary decomposition of $\mathcal{G}_{4}^{*}$ using the routine primdecGTZ in the primdec.LIB library. The result is that $\mathcal{G}_{4}^{*}=\mathcal{N} \cap \mathcal{R}$ where $\mathcal{R}$ is a prime ideal, $\mathcal{N}$ is primary but not prime, and $\sqrt{\mathcal{N}}=\left\langle B, C, D, 5 A-\omega^{*} B\right\rangle$. These generators leads to

$$
\mathbf{V}_{\mathbb{R}}(\mathcal{N})=\mathbf{V}_{\mathbb{R}}(\sqrt{\mathcal{N}})=\left\{\nu \in \mathbb{R}^{4}: B=C=D=5 A-\omega^{*} B=0\right\} .
$$

In summary, since the minimal basis of $\mathcal{G}_{4}$ has cardinality 4 , Theorem 14(ii) tell us that the cyclicity of the center at the origin, only allowing perturbations inside family (15) keeping $\omega=\omega^{*} \in \Omega$ constant, is at most 3 for the parameters belonging to $\left(\mathbf{V}_{\mathscr{C}} \backslash \mathbf{V}_{\mathbb{R}}(\mathcal{N})\right) \cap \mathcal{E}=\left\{\left(\omega^{*}, \nu\right) \in \mathbf{V}_{\mathscr{C}} \cap \mathcal{E}: C \neq 0\right\}$. Notice that this bound is not sharp due to statement (i) of Theorem 19 which is stronger than the former claim.

\subsection{Proof of Theorem 20.}

Proof. We claim that the origin is a nilpotent center if and only if $g$ is an odd function, that is $g(-x ; \nu)=g(x ; \nu)$, in which case they are time-reversible centers with respect to the symmetry $(x, y, t) \mapsto(-x, y,-t)$. The reader can check the claim using for instance the criterium explained in Remark 31 taking into account that $f$ is linear. Therefore the center variety $\mathbf{V}_{\mathscr{C}}$ is (19).

The first integrability focus quantities reduced modulo the ideal generated by the previous one in the ring $\mathbb{R}(\omega)[\nu]$, up to positive multiplicative constants, are

$$
\tilde{\eta}_{j}(\omega, \nu)=\omega \nu_{2(j+1)} \text { for all } j=1, \ldots, m,
$$

hence $\mathbf{V}_{\mathscr{C}} \cap \mathcal{E}=\mathbf{V}_{\mathbb{R}}\left(\mathcal{G}_{m}\right) \cap \mathcal{E}$ and the $m$ is as stated in the theorem. From here we see that the maximum order of a focus at the origin is $m$ and from (59) that the $\tilde{\eta}_{j}$ are independent and can be adjusted with total freedom, hence the bound $m-1$ of the focus cyclicity can be reached proving statement (ii). 
We are going to study the cyclicity of the center at the origin of family (17) by means of Theorem 15 . The center variety only has one irreducible component $C=\mathbf{V}_{\mathscr{C}}$ of $\operatorname{codim}(C)=m$. We consider the map $(\omega, \nu) \mapsto G_{m}(\omega, \nu)=$ $\left(\tilde{\eta}_{1}(\omega, \nu), \ldots, \tilde{\eta}_{m}(\omega, \nu)\right)$. Due to (59) it is easy to check that the $m \times(d-2)$ Jacobian matrix $d_{P} G_{m}$ has full rank (that is, $\operatorname{rank}\left(d_{P} G_{m}\right)=m$ ) at any point $P \in C \cap \mathcal{E}$, and therefore statement (i) follows by Theorem 15(ii).

Remark 35. For family $(17),\left\{\tilde{\eta}_{1}(\omega, \nu), \ldots, \tilde{\eta}_{m}(\omega, \nu)\right\}$ is a minimal bases of $\mathcal{G}_{m}$ of cardinality $m$ since in this example the $\tilde{\eta}_{j}$ lie in $\mathbb{R}[\omega, \nu]$ (look at (59)) rather than in the bigger ring $\mathbb{R}(\omega)[\nu]$ as usual. Also $\mathbf{V}_{\mathbb{R}}(\mathcal{G}) \cap \mathcal{E}=\mathbf{V}_{\mathbb{R}}\left(\mathcal{G}_{m}\right) \cap \mathcal{E}$ as we have seen in the proof of Theorem 20. At first glance it seems that family (17) is a good candidate to apply Theorem 14(i) because the monomial ideal $\mathcal{G}_{m}$ is radical, i.e., $\mathcal{G}_{m}=\sqrt{\mathcal{G}_{m}}$. But first we need to rely on Proposition 11 and, moving to the complex setting, we define the map $F: \mathbb{C}^{d-2} \rightarrow \mathbb{C}^{m}$ via $(\omega, \nu) \mapsto F(\omega, \nu)=\left(\tilde{\eta}_{1}(\omega, \nu), \ldots, \tilde{\eta}_{m}(\omega, \nu)\right)$. We see in the proof of Theorem 20 that $F$ is the complexification of the map $G_{m}$ and then $\operatorname{rank}\left(d_{P} F\right)=m$ at any point $P \in \mathbf{V}_{\mathbb{C}}\left(\mathcal{G}_{m}\right) \backslash \Sigma$ where $\Sigma=\left\{(\omega, \nu) \in \mathbb{C}^{d-2}\right.$ : $\omega=0\} \varsubsetneqq \mathbf{V}_{\mathbb{C}}\left(\mathcal{G}_{m}\right)$ is a hyperplane in $\mathbb{C}^{d-2}$. Unfortunately, in this example $\Sigma$ is a complete irreducible component of the variety $\mathbf{V}_{\mathbb{C}}\left(\mathcal{G}_{m}\right)$ because $\mathbf{V}_{\mathbb{C}}\left(\mathcal{G}_{m}\right)=\Sigma \cup \mathbf{V}_{\mathscr{C}}$. Hence the Zariski closure $\overline{\mathbf{V}_{\mathbb{C}}\left(\mathcal{G}_{m}\right) \backslash \Sigma}=\mathbf{V}_{\mathscr{C}} \neq \mathbf{V}_{\mathbb{C}}\left(\mathcal{G}_{m}\right)$ and we cannot apply Proposition 11.

\subsection{Proof of Theorem 21.}

Proof. Taking into account that (20) is time-reversible with respect to the symmetry $(x, y, t) \mapsto(-x, y,-t)$ when the parameters are in $C^{\text {sym }}$ it follows that $(20)$ has a center at the origin when the parameters lie in $C^{\mathrm{sym}} \cap \mathcal{E}$.

The first integrability focus quantities reduced modulo the ideal generated by the previous one in the ring $\mathbb{R}(\omega)[\nu]$, up to positive multiplicative constants, are

$$
\tilde{\eta}_{1}(\omega, \nu)=-\omega \nu_{1}, \quad \tilde{\eta}_{2}(\omega, \nu)=\left(\omega^{2}-7\right) \nu_{4} .
$$

The center cyclicity at the origin with parameters on the irreducible component $C^{\text {sym }}$ with $\operatorname{codim}\left(C^{\text {sym }}\right)=2$ will be analyzed with Theorem 15 . We define the map $(\omega, \nu) \mapsto G_{2}(\omega, \nu)=\left(\tilde{\eta}_{1}(\omega, \nu), \tilde{\eta}_{2}(\omega, \nu)\right)$ whose linearization at any point $P \in \mathbb{R}^{6}$ is

$$
d_{P} G_{2}=\left(\begin{array}{cccccc}
-\nu_{1} & -\omega & 0 & 0 & 0 & 0 \\
2 \omega \nu_{4} & 0 & 0 & 0 & \omega^{2}-7 & 0
\end{array}\right) .
$$

Then $\operatorname{rank}\left(d_{P} G_{2}\right)=2$ at any point $P \in C^{\mathrm{sym}} \cap \mathcal{E}$ except when $\omega^{2}=7$. Hence the theorem follows by Theorem 15(ii).

\section{REFERENCES}

[1] A. Algaba, C. García and J. Giné, Nilpotent centres via inverse integrating factors, European J. Appl. Math. 27 (2016), 781-795.

[2] A. Algaba, C. García, J. Giné and J. Llibre, The center problem for $\mathbb{Z}_{2}$-symmetric nilpotent vector fields, Preprint. Universitat de Lleida.

[3] A. Algaba, C. García And M. Reyes, Existence of an inverse integrating factor, center problem and integrability of a class of nilpotent systems, Chaos Solitons Fractals 45 (2012), 869-878.

[4] M.J. Álvarez And A. Gasull, Monodromy and stability for nilpotent critical points, Internat. J. Bifur. Chaos Appl. Sci. Engrg. 15 (2005), 1253-1265.

[5] M.J. Álvarez and A. Gasull, Generating limit cycles from a nilpotent critical point via normal forms, J. Math. Anal. Appl. 318 (2006), 271-287. 
[6] A. Andreev, Investigation on the behaviour of the integral curves of a system of two differential equations in the neighborhood of a singular point, Translations Amer. Math. Soc. 8 (1958) 187-207.

[7] N.N. BAUtin, On the number of limit cycles which appear with the variations of the coeffcients from an equilibrium point of focus or center type, AMS Translations-Series 1, 5, 1962, 396-413 [Russian original: Math. Sbornik, 30, 1952, 181-196.

[8] J. Chavarriga, H. Giacomini, J. Giné and J. Llibre, Local analytic integrability for nilpotent centers. Ergodic Theory Dynam. Systems 23 (2003), no. 2, 417-428.

[9] L. A. Cherkas, Conditions for a Liénard equation to have a centre, Differential Equations 12 (1976), 201-206.

[10] C. Christopher. Estimating limit cycles bifurcations from centers. In: Trends in Mathematics, Differential Equations with Symbolic Computations, 23-36. Basel: Birkhäuser-Verlag, 2005.

[11] C. Christopher And C. LI, Limit cycles of differential equations. Advanced Courses in Mathematics. CRM Barcelona. Birkhuser Verlag, Basel, 2007.

[12] W. Decker, G.M. Greuel, G. Pfister and Schönemann. Singular 4-1-1. A computer algebra system for polynomial computations. http://www.singular.uni-kl.de, 2018.

[13] W. Decker, G. Pfister, H. Schönemann, and S. Laplagne. primdec.lib. Singular 4-1-1 library for computing primary decomposition and radical of ideals (2018).

[14] J. ÉCALLE, Introduction aux fonctions analysables et preuve constructive de la conjecture de Dulac, Actualités Mathématiques, Hermann, Paris, 1992.

[15] B. Ferčec, V. Levandovskyy, V.G. Romanovski, and D.S. Shafer, Bifurcation of critical periods of polynomial systems, J. Differential Equations 259 (2015), 3825-3853.

[16] I.A. García, Formal inverse integrating factors and the nilpotent center problem, Internat. J. Bifur. Chaos Appl. Sci. Engrg. 26 (2016), 1650015.

[17] I.A. García, Cyclicity of some symmetric nilpotent centers, J. Differential Equations 260 (2016), 5356-5377.

[18] I.A. García, H. Giacomini and M. Grau, The inverse integrating factor and the Poincaré map, Trans. Amer. Math. Soc., 362 (2010), 3591-3612.

[19] I.A. García, H. Giacomini and M. Grau, Generalized Hopf bifurcation for planar vector fields via the inverse integrating factor, J. Dyn. Differ. Equ. 23 (2011), 251-281.

[20] I.A. García, S. Maza AND D.S. Shafer, Cyclicity of polynomial nondegenerate centers on center manifolds. To appear in J. Differential Equations.

[21] I.A. García AND D.S. Shafer, Cyclicity of a class of polynomial nilpotent center singularities, Discrete Contin. Dyn. Syst. 36 (2016), 2497-2520.

[22] A. Gasull And J. Torregrosa, Center problem for several differential equations via Cherkas' method, J. Math. Anal. Appl. 228 (1998), 322-343.

[23] M. Golubitsky and D.G. Schaeffer, Singularities and Groups in Bifurcation Theory: Volume I. Applied Mathematical Sciences. Springer. 1984.

[24] Y. Ilyashenko, Finiteness Theorems for Limit Cycles, Transl. Math. Monogr., vol. 94, Amer. Math. Soc., Providence, RI, 1991.

[25] V. Levandovskyy, A. Logar, and V.G. Romanovski, The cyclicity of a cubic system, Open. Syst. Inf. Dyn. 16 (2009) 429-439.

[26] Y. LiU And J. Li, New study on the center problem and bifurcations of limit cycles for the Lyapunov system (I), Internat. J. Bifur. Chaos 19 (2009), 3791-3801.

[27] Y. LiU And J. LI, New study on the center problem and bifurcations of limit cycles for the Lyapunov system (II), Internat. J. Bifur. Chaos 19 (2009), 3087-3099.

[28] Y. LiU AND J. LI, On third-order nilpotent critical points: Integral factor method, Internat. J. Bifur. Chaos 21 (2011), 1293-1309.

[29] A. M. Lyapunov, Stability of motion. Mathematics in Science and Engineering, Vol 30. Academic Press, New York-London (1966).

[30] J.E. Marsden, AND T.S. Ratiu, Introduction to Mechanics and Symmetry. A basic exposition of classical mechanical systems. Texts in Applied Mathematics, 17. Springer- Verlag, (1994) New York.

[31] V. G. Romanovski And D. S. ShafeR, The center and cyclicity problems: a computational algebra approach. Birkhäuser Boston, Inc., Boston, MA, 2009.

[32] E. StrózYNA \& H. ŻOŁA̧DEK, The analytic and formal normal form for the nilpotent singularity, J. Differential Equations 179 (2002), 479-537. 
[33] J. Su, J. YANG And M. HAN, Hopf bifurcation of Liénard systems by perturbing a nilpotent center, Internat. J. Bifur. Chaos 22 (2012), 1250203, 7 pp.

Departament de Matemàtica, Universitat de Lleida, Avda. Jaume II, 69, 25001 Lleida, SPAIN

E-mail address: garcia@matematica.udl.cat 